\title{
EVOLUTION OF DUST AND ICE FEATURES AROUND FU ORIONIS OBJECTS ${ }^{1}$
}

\author{
S. P. Quanz, Th. Henning, J. Boumman, R. van Boekel, A. Juhász, and H. Linz \\ Max-Planck-Institut für Astronomie, Heidelberg D-69117, Germany; quanz@mpia.de \\ K. M. Pontoppidan ${ }^{2}$ \\ California Institute of Technology, Division of Geological and Planetary Sciences, Pasadena, CA 91125 \\ AND \\ F. LAHUIS \\ Leiden Observatory, Leiden University, 2300 RA Leiden, Netherlands; and SRON Netherlands \\ Institute for Space Research, Groningen, Netherlands \\ Received 2007 May 11; accepted 2007 June 24
}

\begin{abstract}
We present spectra for a sample of 14 FUors and two T Tauri stars observed with Spitzer or ISO. Based on the appearance of the $10 \mu \mathrm{m}$ silicate feature, we define two categories of FUors. Objects showing the silicate feature in absorption (Category 1) are still embedded in a circumstellar envelope. The shape of the silicate bands is in good agreement with typical dust compositions of the ISM. Only one object appears too rich in amorphous pyroxene dust, but a superposed emission feature can explain the observed shape. We derive optical depths and extinction values from the silicate and additional ice bands. Particularly, the analysis of the $\mathrm{CO}_{2}$ ice band at $15.2 \mu \mathrm{m}$ allows us to search for evidence for ice processing and to constrain whether the absorbing material is physically linked to the central object. For objects showing the silicate band in emission (Category 2), we argue that the feature comes from the surface layer of accretion disks. We find evidence that grain growth has already taken place within the disks, but no clear indications for crystallization are present. We discuss how these observations fit into the picture of a young and active accretion disk. Finally, a framework is proposed how the two categories of FUors can be understood in a general paradigm of the evolution of young, low-mass stars. As one object (Parsamian 21) shows PAH emission features typical for evolved stars, we question its status as a FUor. Additionally, two spectra (RNO 1B and L1551 IRS 5) show [Fe II] emission lines that are attributed to hot, dense, or shocked material related to outflows.
\end{abstract}

Subject headings: accretion, accretion disks - circumstellar matter — dust, extinction — stars: formation stars: individual (FU Ori) — stars: pre-main-sequence

Online material: color figures

\section{INTRODUCTION}

By means of mid-infrared (MIR) spectroscopy, gaseous and solid-state features have been observed and analyzed in a variety of astronomical environments. While ground-based observations are restricted to certain atmospheric windows (e.g., the $N$ band around $10 \mu \mathrm{m}$ ), spectrographs on board space-borne telescopes, such as the Infrared Space Observatory (ISO) and the Spitzer Space Telescope, enable us to study a broad wavelength range from the near-infrared (NIR) to well beyond $30 \mu \mathrm{m}$. Those instruments fostered and revolutionized our understanding in numerous astronomical fields of research. In particular, the star formation community benefitted from studies based on data from the space telescopes. The composition of dust grains and their evolution (e.g., grain growth and crystallization) in protoplanetary disks was analyzed to great extent in young, intermediate-mass Herbig Ae/Be stars (HAeBes; e.g., Bouwman et al. 2001; Meeus et al. 2001; Acke \& van den Ancker 2004; van Boekel et al. 2005), but also in the young, less massive T Tauri stars (e.g., Forrest et al. 2004; Kessler-Silacci et al. 2006; Sargent et al. 2006). In addition,

\footnotetext{
${ }^{1}$ Based on observations with ISO, an ESA project with instruments funded by ESA Member States (especially the PI countries: France, Germany, the Netherlands, and the United Kingdom) and with the participation of ISAS and NASA. This work is based in part on observations made with the Spitzer Space Telescope, which is operated by the Jet Propulsion Laboratory, California Institute of Technology under a contract with NASA.

2 Hubble Fellow.
}

the ice and dust features of younger and more deeply embedded objects were studied (e.g., Watson et al. 2004), and the ice inventory of molecular clouds was investigated (e.g., Knez et al. 2005; Bergin et al. 2005).

One special subgroup of young low-mass objects are FU Orionis objects (FUors), named after the prototype FU Ori. For most objects of this group evidence was found for a tremendous outburst in optical or NIR light over short timescales (months to years) followed by a decline in luminosity typically over several decades. Other objects were included in this group as they shared peculiar spectral features, e.g., CO band head absorption in the NIR and a changing spectral type with wavelength. Most observational data can be well explained with highly active accretion disks surrounding these objects, possibly fed with fresh material from a remnant envelope (for a review see Hartmann \& Kenyon 1996). Observations in the MIR are thus ideally suited to probe the dusty component of the circumstellar material of these objects, contained either in the accretion disks or, additionally, in the envelopes. Recent findings from NIR and MIR interferometers support the presence of accretion disks several tens of AU in size (Quanz et al. 2006; Malbet et al. 2005; Millan-Gabet et al. 2006). However, while the spectral energy distribution (SED) and the NIR and MIR visibilities of FU Ori itself can be sufficiently explained by a simple accretion disk model (Quanz et al. 2006), Millan-Gabet et al. (2006) found that accretion disks alone cannot reproduce the SED and observed low $K$-band visibilities of V1057 Cyg, V1515 Cyg, and Z CMa-SE simultaneously. They concluded that 
TABLE 1

JouRnAL OF ISO SWS OBSERVATIONS

\begin{tabular}{|c|c|c|c|c|c|c|}
\hline Object & $\begin{array}{c}\text { R.A. } \\
(\mathrm{J} 2000.0)\end{array}$ & $\begin{array}{c}\text { Decl. } \\
(\mathrm{J} 2000.0)\end{array}$ & AOT/Speed & $\begin{array}{l}\text { Time on Target } \\
\text { (s) }\end{array}$ & $\begin{array}{c}y / z \text { Offset } \\
(\operatorname{arcsec})\end{array}$ & Date \\
\hline \multirow[t]{5}{*}{ 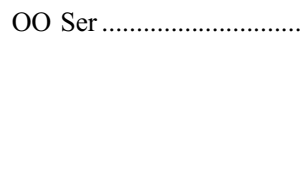 } & 182949.05 & +011619.2 & SWS01/1 & 1062 & $\ldots$ & 1996 Apr 14 \\
\hline & 182949.08 & +011619.8 & SWS01/1 & 1140 & $4 / 3$ & 1996 Oct 24 \\
\hline & 182949.05 & +011619.2 & SWS01/2 & 1140 & $\ldots$ & 1997 Mar 08 \\
\hline & 182949.05 & +01 1619.2 & SWS01/3 & 3454 & $\ldots$ & 1997 Apr 12 \\
\hline & 182949.09 & +01 1619.8 & SWS01/3 & 3454 & $\ldots$ & 1997 Sep 22 \\
\hline RNO 1B & 003646.24 & +632854.3 & SWS01/2 & 1912 & $\ldots$ & 1996 Aug 27 \\
\hline 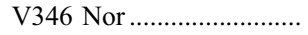 & 163232.05 & -445528.9 & SWS01/2 & 1912 & $\ldots$ & 1996 Aug 31 \\
\hline 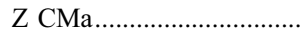 & 070343.17 & -113306.6 & SWS01/2 & 3454 & $3 / 3$ & 1997 Nov 07 \\
\hline 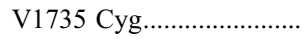 & 214720.60 & +473204.9 & SWS01/2 & 1912 & $4 / 4$ & 1996 Aug 06 \\
\hline 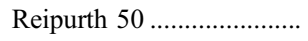 & 054017.89 & -072729.3 & SWS01/3 & 3454 & $5 / 5$ & 1997 Oct 13 \\
\hline L1551 IRS $5 \ldots \ldots \ldots \ldots \ldots \ldots$ & 043134.06 & +180804.8 & SWS01/4 & 6538 & $\ldots$ & 1997 Sep 06 \\
\hline
\end{tabular}

NoтEs.-Units of right ascension are hours, minutes, and seconds, and units of declination are degrees, arcminutes, and arcseconds. The coordinates denote the pointing position of the telescope as saved in the header of the data files. The speed of the observations, the time spent on the target, and possible pointing offsets in the telescope's $y$-and $z$-axes are given as well. The offsets were corrected for during the data reduction applying the measured beam profiles along the different axes.

additional uncorrelated flux is required, possibly arising due to scattering by large dusty envelopes.

While Lorenzetti et al. (2000) presented far-infrared spectroscopic data for six FUors observed with ISO LWS, a dedicated MIR study of a larger sample of FUors is still missing. Larsson et al. (2000) showed the ISO Short Wavelength Spectrograph (SWS) spectra for six FUors for comparison, but no analysis was carried out. The ISO data for Z CMa were presented in publications related to HAeBes by Acke \& van den Ancker (2004) and Elia et al. (2004) as Z CMa presumably is a binary system consisting of a Herbig star and a FUor. White et al. (2000) used the ISO observations of L1551 IRS 5 as input for a radiative transfer model of this source. Hanner et al. (1998) discussed ground-based 8-13 $\mu \mathrm{m}$ spectra for four FUors (FU Ori, V1515 Cyg, V1057 Cyg, V1735 Cyg) and fitted a simple dust model to the data to check whether silicate particles from the interstellar medium (ISM) can reproduce the observed features. Schütz et al. (2005) published additional ground-based data for another four objects in the same wavelength regime. As three objects ( Z CMa, V346 Nor, V883 Ori) showed the $10 \mu \mathrm{m}$ feature in absorption, they inferred the optical depth from fitting an ISM dust model to the spectra. The fourth object (Bran 76, alias BBW 76) was not analyzed in greater detail. Polomski et al. (2005) presented data on RNO 1B, Z CMa, and Parsamian 21 and derived dust temperatures and optical depths.

To our knowledge, the first MIR spectra of FUors observed with Spitzer were presented in Green et al. (2006). The main focus of this publication was an accurate SED modeling of FU Ori, V1515 Cyg, and V1057 Cyg. Spectral solid-state features were not analyzed in greater detail. The spectrum of V346 Nor was presented for comparison. In Quanz et al. (2006) the first detailed dust composition modeling for the FU Ori spectrum was presented and evidence for grain growth in the accretion disk was found. The Spitzer spectra of RNO 1B/1C were shown in Quanz et al. (2007). However, the dust composition was not yet analyzed in detail.

In this paper we compile MIR spectra for $14 \mathrm{FU}$ Orionis objects observed with $I S O$ and/or Spitzer. As up to now only $\sim 20$ FUors or FUor candidates are known, this is the largest sample of these objects analyzed so far in a single MIR study. Part of the data have not been published before. For objects where the spectra show a sufficient signal-to-noise ratio, the dust and ice composition of the circumstellar material is investigated.

\section{OBSERVATIONS AND DATA REDUCTION}

The MIR spectra we present in this paper are compiled from the archives of the $I_{S O}{ }^{3}$ and the Spitzer ${ }^{4}$ satellites. The SWS on board $I S O$ consisted of two nearly independent grating spectrometers with a spectral resolution between 1000 and 2500 (depending on the band and order) and covering a wavelength range from 2.4 to $12.0 \mu \mathrm{m}$ and from 12.0 to $45.0 \mu \mathrm{m}$, respectively. While the field of view (FOV) for the shorter wavelength regime was $14^{\prime \prime} \times 20^{\prime \prime}$, the FOV for the longer wavelength range was $14^{\prime \prime} \times$ $27^{\prime \prime}$, with the exception of the wavelength range between 28.9 and $45.0 \mu \mathrm{m}$, which had an FOV of $20^{\prime \prime} \times 33^{\prime \prime}$. With ISO SWS seven objects classified as FUors were observed between 1996 April and 1997 October. For one object (OO Ser) data were taken at five different epochs, documenting a decay in luminosity over a few months. Table 1 summarizes the ISO observations with object names, coordinates, ISO SWS observing mode and scan speed, integration time on target, possible pointing offsets (see below), and the date of the observation. For the data reduction Highly Processed Data Products (HPDPs) or SWS Auto Analysis Results (AARs) were downloaded from the ISO archive for speed 1 and 2 or speed 3 and 4 observations, respectively. With the OSIA software package ${ }^{5}$ (ver. 4.0) the following reduction steps were carried out: For each object the spectra from the ISO SWS up and down scans were flat-fielded and rebinned. After sigma clipping, the speed 3 and speed 4 spectra were defringed. This procedure was not required for the speed 1 and speed 2 data as the HPDPs are already defringed. Finally, the spectra from the up and down scans were combined and rebinned to a spectral resolution of 100 . In case the resulting spectrum showed signs of a pointing offset (e.g., aperture jumps), a correction based on the measured beam profiles along the different axes was applied to the raw data and the data reduction was repeated. The applied offsets are listed in Table 1.

The Spitzer observations are summarized in Table 2. The IRS on board Spitzer offers a short-wavelength, low-resolution module (SL) covering the wavelength range between 5.2 and $14.5 \mu \mathrm{m}$ and a short-wavelength, high-resolution module (SH) going from

\footnotetext{
3 The $I S O$ archive can be accessed via http://www.iso.vilspa.esa.es/ida/.

${ }_{5}^{4}$ See http://ssc.spitzer.caltech.edu/archanaly/archive.html.

5 See http://sws.ster.kuleuven.ac.be/osia/.
} 
TABLE 2

Journal of Spitzer IRS ObSERVATIONS

\begin{tabular}{|c|c|c|c|c|c|}
\hline & R.A. & Decl. & & Integration Time & \\
\hline Object & $(\mathrm{J} 2000.0)$ & $(\mathrm{J} 2000.0)$ & AOR & (s) & Date \\
\hline Bran 76 (BBW 76) & 075035.52 & -330624.12 & 3571200 & 12 (SL, LL) & 2004 Apr 14 \\
\hline FU Ori. & 054522.39 & +0904 12.5 & 3569920 & $12(\mathrm{SL}, \mathrm{SH}, \mathrm{LH})$ & 2004 Mar 04 \\
\hline L1551 IRS 5 & 043134.08 & +180804.92 & 3531776 & $6(\mathrm{SL}, \mathrm{SH}, \mathrm{LH})$ & 2004 Mar 04 \\
\hline Parsamian 21 (HBC 687) ...................... & 192900.72 & +093847.11 & 5039872 & $\begin{array}{c}36(\mathrm{SL}) \\
48(\mathrm{SH}, \mathrm{LH})\end{array}$ & 2004 Apr 18 \\
\hline 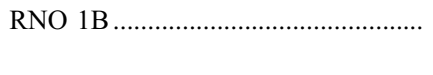 & 003646.34 & +632853.76 & 6586624 & $\begin{array}{c}36(\mathrm{SL}) \\
60(\mathrm{SH}, \mathrm{LH})\end{array}$ & 2004 Jan 07 \\
\hline RNO 1C & 003646.89 & +632858.44 & 6586624 & $\begin{array}{c}36(\mathrm{SL}) \\
60(\mathrm{SH}, \mathrm{LH})\end{array}$ & 2004 Jan 07 \\
\hline 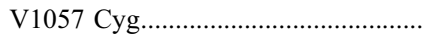 & 205853.76 & +441528.44 & 3570176 & $12(\mathrm{SL}, \mathrm{SH}, \mathrm{LH})$ & 2003 Dec 15 \\
\hline 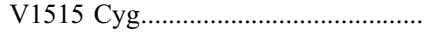 & 202348.00 & +421225.56 & 3570432 & $12(\mathrm{SL}, \mathrm{SH}, \mathrm{LH})$ & 2004 May 11 \\
\hline \multirow[t]{3}{*}{ 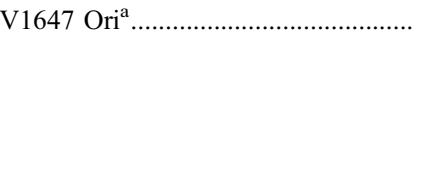 } & 054613.13 & -000605.21 & 12261120 & $\begin{array}{c}12(\mathrm{SL}) \\
24(\mathrm{SH}, \mathrm{LH})\end{array}$ & 2004 Oct 20 \\
\hline & 054613.15 & -000604.41 & 11569920 & $\begin{array}{c}48(\mathrm{SL}) \\
484(\mathrm{SH}), 240(\mathrm{LH})\end{array}$ & 2005 Mar 11 \\
\hline & 054613.14 & -000604.69 & 12644096 & $12(\mathrm{SL}, \mathrm{LL})$ & 2005 Mar 24 \\
\hline 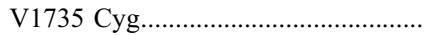 & 214720.6 & +473200.7 & 3570944 & $12(\mathrm{SL}, \mathrm{SH}, \mathrm{LH})$ & 2003 Dec 17 \\
\hline 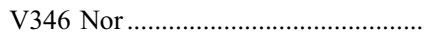 & 163232.1 & -445528.6 & 3570688 & $12(\mathrm{SL}, \mathrm{SH}, \mathrm{LH})$ & $2004 \mathrm{Feb} 27$ \\
\hline HL Tau & 043138.4 & +18 1357.9 & 3531776 & $6(\mathrm{SL}, \mathrm{SH}, \mathrm{LH})$ & 2004 Mar 04 \\
\hline XZ Tau AB & 043140.1 & +18 1357.4 & 3531776 & $6(\mathrm{SL}, \mathrm{SH}, \mathrm{LH})$ & 2004 Mar 04 \\
\hline
\end{tabular}

Notes.- Units of right ascension are hours, minutes, and seconds, and units of declination are degrees, arcminutes, and arcseconds. The coordinates denote the average slit position of the low-resolution spectrograph as computed by the onboard software. The AOR of the observations, the time spent on the target for the different modules, and the observation date are given as well.

${ }^{\text {a }}$ The object was observed at three different epochs.

9.9 to $19.6 \mu \mathrm{m}$. The corresponding slit sizes are $\approx 3.6^{\prime \prime} \times 136.0^{\prime \prime}$ (including both SL orders and an additional bonus segment connecting both orders) and $4.7^{\prime \prime} \times 11.3^{\prime \prime}$, respectively. For the longer wavelength part a long-wavelength, low-resolution module (LL) ranging from 14.0 to $38.0 \mu \mathrm{m}$ and a long-wavelength, highresolution model (LH) covering the regime from 18.7 to $37.2 \mu \mathrm{m}$ are available. The slits sizes are $\approx 10.5^{\prime \prime} \times 360.0^{\prime \prime}$ (including both LL orders and an additional bonus segment connecting both orders) and $11.1^{\prime \prime} \times 22.3^{\prime \prime}$, respectively. Both low-resolution modules (SL and LL) offer a spectral resolution between 64 and 128 (depending on the wavelength), while the high-resolution modules both have $\sim 600$. All objects listed in Table 2 were observed with the full wavelength coverage, either with a combination of $\mathrm{SL}+\mathrm{LL}$ or with $\mathrm{SL}+\mathrm{SH}+\mathrm{LH}$. Two objects (HL Tau and XZ Tau) are not classified as FUors but were part of a small Spitzer IRS map including L1551 IRS 5, and data for all three objects could be downloaded simultaneously. Interestingly, XZ Tau is a binary system that recently was found to show EXor-type variations (Coffey et al. 2004), i.e., another type of short-term eruptions of young stars. Thus, a comparison to the FUor data presented here is reasonable. In addition, the data of HL Tau enable us to compare the FUor spectra to that of a well-studied young star with a remnant envelope and a highly inclined accretion disk seen almost edge-on (Close et al. 1997). Part of the Spitzer spectrum of HL Tau was already published in Bergin et al. (2005). The object V1647 Ori was observed three times within a period of roughly 5 months to monitor its brightness as it underwent an eruption in the beginning of 2004. However, to our knowledge, thus far no spectrum has been published.

The data reduction process of the Spitzer data is described in detail in Quanz et al. (2007). However, we should mention that the error bar for each individual spectral point represents the formal standard deviation from the mean flux of at least two independent measurements (two telescope nod positions and possibly several observation cycles). Also taken into account is the formal error of the spectral response function. For details on the method we refer the reader also to Bouwman et al. (2006). We estimate a relative flux calibration error across a spectral order of $\approx 5 \%$ and an absolute calibration error between orders or modules of $\approx 10 \%$. In particular, for the objects RNO 1B and RNO 1C apparent flux density offsets between the SL and the SH part of the spectra, as well as between the short- and long-wavelength parts of the highresolution spectra, are already discussed in Quanz et al. (2007). For the other objects presented here, the discrepancies in the flux densities between the SL and the SH part of the spectrum were $<10 \%$, and we matched the longer wavelength part to the shorter regime by multiplying a scalar factor. Only Bran 76 (also known as BBW 76) showed a larger offset of $\approx 15 \%$ as already mentioned by Green et al. (2006). For Parsamian 21 (HBC 687) we do not show the SH spectrum between 14 and $20 \mu \mathrm{m}$ as the slit of the spectrograph was not centered on the source and significant flux loss occurred for which we could not correct. An additional correction between the $\mathrm{SH}$ and the $\mathrm{LH}$ module of the spectrum was required for XZ Tau, HL Tau, and L1551 IRS 5, where the flux densities of the LH part had to be scaled down by $10 \%-15 \%$. This offset can be explained by the larger aperture of this module, which possibly probed additional large-scale emission from the surroundings of these objects.

\section{RESULTS}

\subsection{General Overview}

Figures 1-8 show the complete sample of spectra. For Bran 76 and the third observation of V1647 Ori only low-resolution Spitzer data were available. To increase the signal-to-noise ratio, all Spitzer LH data and the SH data of V1735 Cyg were smoothed by a factor of 3. Most of the yet remaining spikes in these spectra are not real but rather flux jumps between the different orders of the spectrographs. As mentioned in the introduction of this paper, parts of the data shown here were already published: Green et al. (2006) 

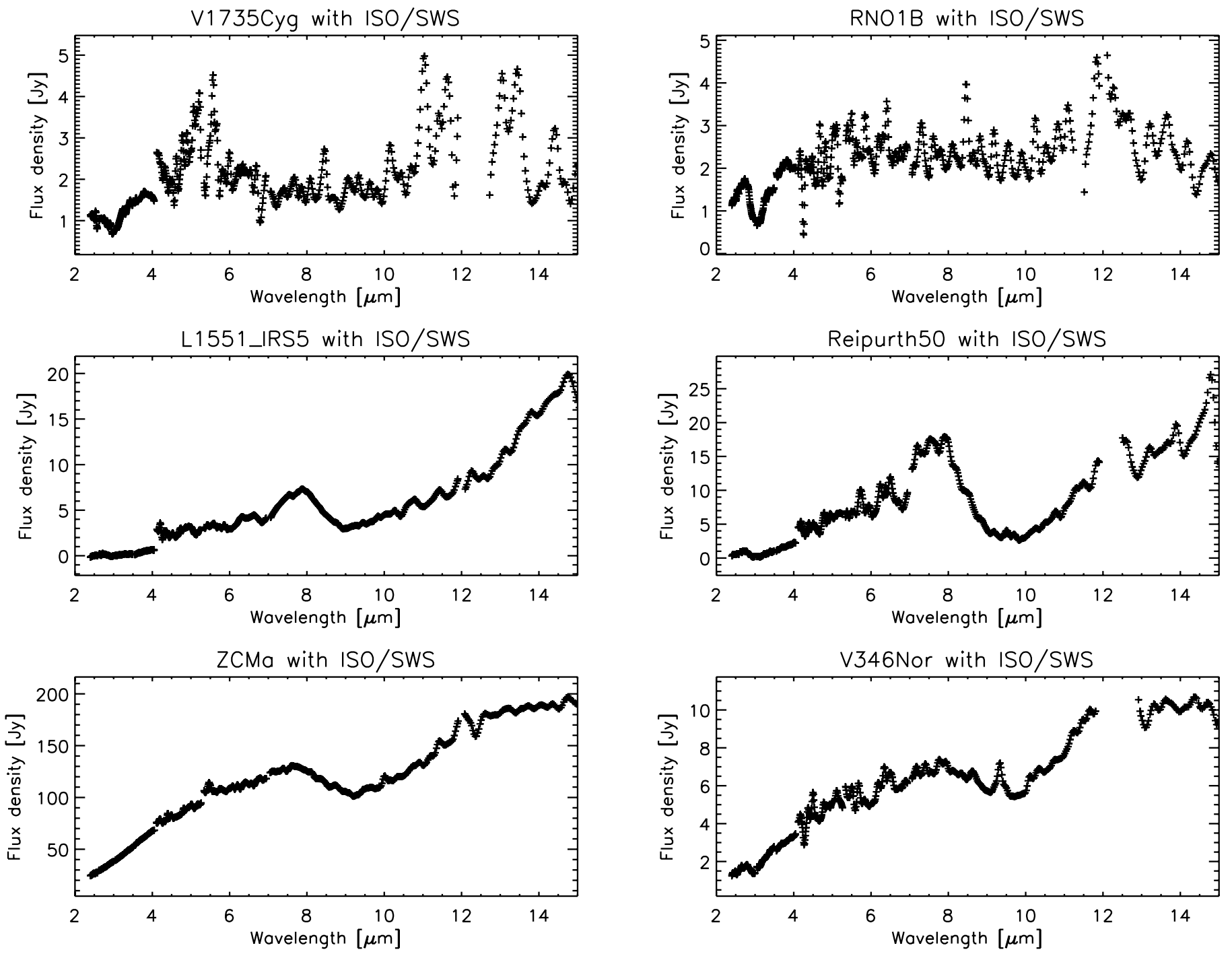

FIG. 1.-ISO SWS spectra in the 2-15 $\mu \mathrm{m}$ range for six of our targets. The objects in the top panels (V1735 Cyg and RNO 1B) suffer from a poor signal-to-noise ratio resulting from a combination of relatively low flux levels and short integration times (Table 1). All substructures in the spectra are not believed to be real.

presented the Spitzer data for FU Ori, V1515 Cyg, V1057 Cyg, Bran 76, and V346 Nor and used disk envelope models to explain the SEDs. The spectrum of HL Tau was shown by Bergin et al. (2005). Schütz et al. (2005) used the ISO data for Z CMa and V346 Nor to compare with their ground-based data. Finally, Larsson et al. (2000) showed the ISO SWS SEDs of the outbursting object OO Ser and used the ISO SWS data for RNO 1B, Z CMa, V1057 Cyg, and V1735 Cyg for comparison.

Unfortunately, for most objects the quality of the ISO SWS data is significantly worse than that of the Spitzer observations. Even after the data have been rebinned to a spectral resolution of 100 , artifacts, i.e., potential emission features that were only detected during either the up or the down scan, remain in the spectra. In Figure 9 we show data for four objects that were observed with both Spitzer and ISO. In particular, for the objects with lower flux levels the noise in the SWS data is significant. The reason for this is the short integration time for most objects, which is reflected in the speed parameter in Table 1. In consequence, the ISO data are mainly used for qualitative statements rather than for quantitative analyses throughout the rest of the paper. Only the ISO data for Z CMa (with a high flux level) and Reipurth 50 (with long integration time) are examined in more detail in one of the con- secutive sections. The data on Reipurth 50 are published for the first time.

From Figures 1-8 it becomes clear that for all objects the flux densities increase toward longer wavelengths, indicative of warm dusty material surrounding all objects. However, Figures 1, 2, 5, and 6 show that there are striking differences within the group of FUors: While some objects show a silicate emission feature in the $10 \mu \mathrm{m}$ region, other objects show deep absorption profiles. Since other spectral features do further support such a differentiation, in the following we distinguish the objects via the behavior of their $10 \mu \mathrm{m}$ feature and discuss the two categories separately in the following subsections. A complete overview of the most prominent spectral features between 3 and $16 \mu \mathrm{m}$ is given in Table 3. If we count V883 Ori, which was observed by Schütz et al. (2005), and disregard XZ Tau and HL Tau, then nine FUors show the silicate feature in absorption while six FUors show silicate emission.

\subsection{Objects with $10 \mu \mathrm{m}$ Emission}

\subsubsection{Qualitative Analysis of the $10 \mu \mathrm{m}$ Region}

In Figure 10 we compiled all objects showing signs of silicate emission in the $10 \mu \mathrm{m}$ band. To subtract the underlying continuum, 

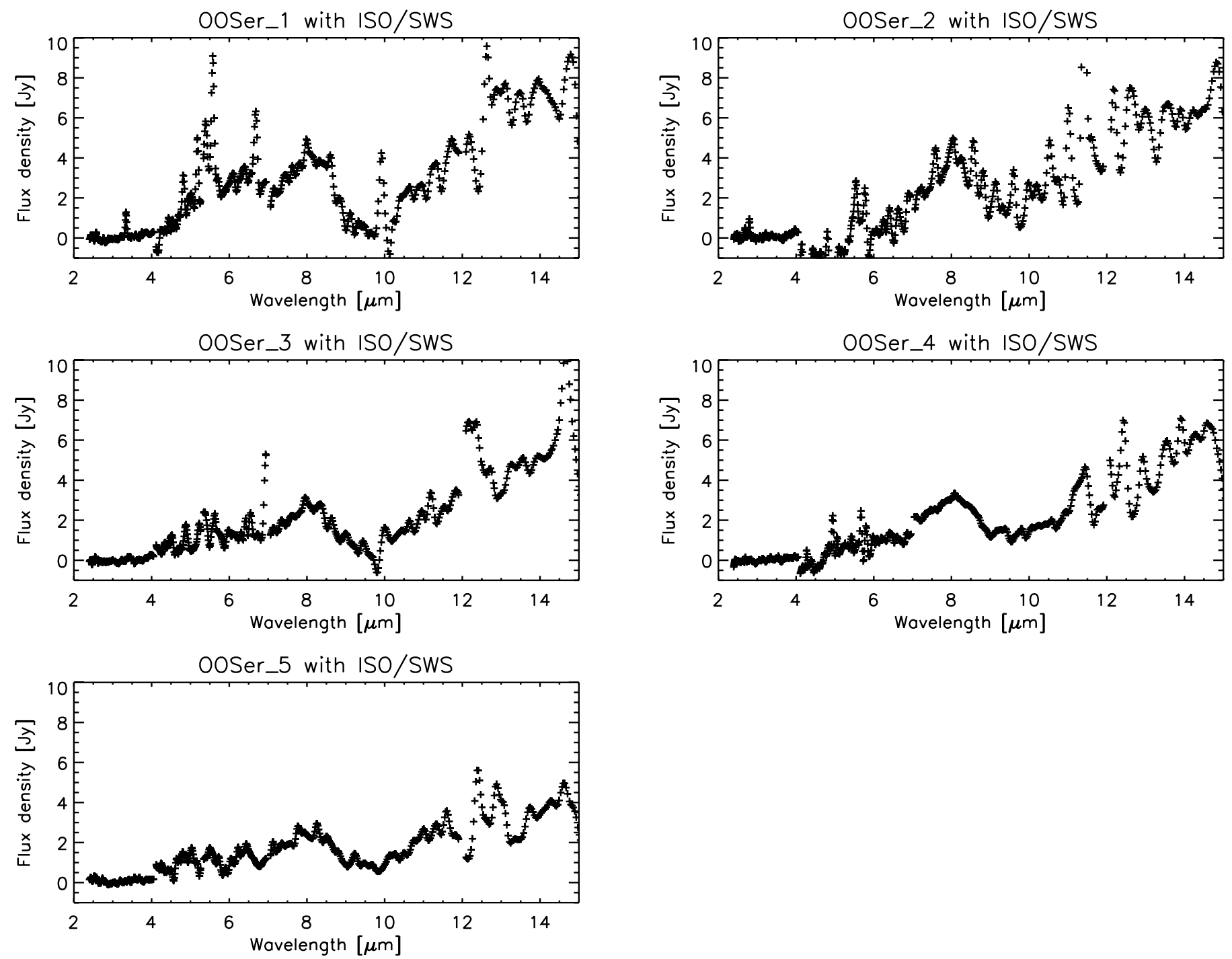

FIG. 2.-Same as Fig. 1, but for the object OO Ser, which was observed at five different epochs. As shown in Table 1, the integration times of the observations were increased during the five epochs. However, as the object faded significantly over this period, the signal-to-noise ratio could not be improved, and it is difficult to identify spectroscopic features in the data apart from the silicate absorption profile seen in all data sets.

we fitted a polynomial of first order to the flux at 8 and $13 \mu \mathrm{m} .^{6}$ As we are thus far only interested in a qualitative comparison among the various objects, the exact shape of the continuum is not important and higher order polynomial fits did not alter the results relative to each other. For comparison we overplot the silicate emission feature of typical ISM dust grains, scaled to the observed spectra (Figure 10, dashed lines; Kemper et al. 2004). We note that the spectrum of each object is shown twice: while the first plot shows the observed spectrum of the source, the second one shows the dereddened spectrum. For the dereddening we compiled optical extinction values from the literature (see caption of Fig. 10) and used the extinction law described in Mathis (1990) to derive corresponding extinction values for the MIR. It is assumed that all of the extinction is caused by material in the line of sight toward the objects and that no self-shadowing effect (e.g., by an inclined accretion disk with a certain flaring angle) is present. The continuum was fitted for the dereddened spectra separately. It shows that for most objects the extinction is not negligible and that it can have substantial influence on the shape

\footnotetext{
${ }^{6}$ For Parsamian 21 we had to shift the left point of the fit to $6.6 \mu \mathrm{m}$ due to the special shape of the spectrum (see below).
}

of the silicate feature (see, e.g., V1057 Cyg and V1647 Ori). For a fair comparison it thus seems reasonable to evaluate the dereddened and not the observed spectra.

At first glance, when comparing the spectra, it is noteworthy that all emission features differ from the typical shape of the typical ISM dust feature. Green et al. (2006) stated that the emission peak for the first four objects in Figure 10 was close to $9.5 \mu \mathrm{m}$ and that the dust features appeared to be pristine. ${ }^{7}$ We find that all features peak longward of $9.7 \mu \mathrm{m}$ (the typical peak position for ISM type dust; Kemper et al. 2004) and that they show additional flux excess compared to the ISM feature at even longer wavelengths. This indicates that dust grain processing has already set in. Furthermore, it should be noted that the spectrum of Parsamian 21 looks significantly different compared to the others. The most prominent characteristics are strong emission bands around $8.2 \mu \mathrm{m}$ and probably also around $11.3 \mu \mathrm{m}$ from polycyclic aromatic hydrocarbons (PAHs). Polomski et al. (2005) already suspected the existence of PAH emission in the spectrum of this source based

\footnotetext{
${ }^{7}$ It should be mentioned that Green et al. (2006) determined the underlying continuum from a fit to the $6-8 \mu \mathrm{m}$ region and not as we did from fitting a straight line between 8 and $13 \mu \mathrm{m}$.
} 

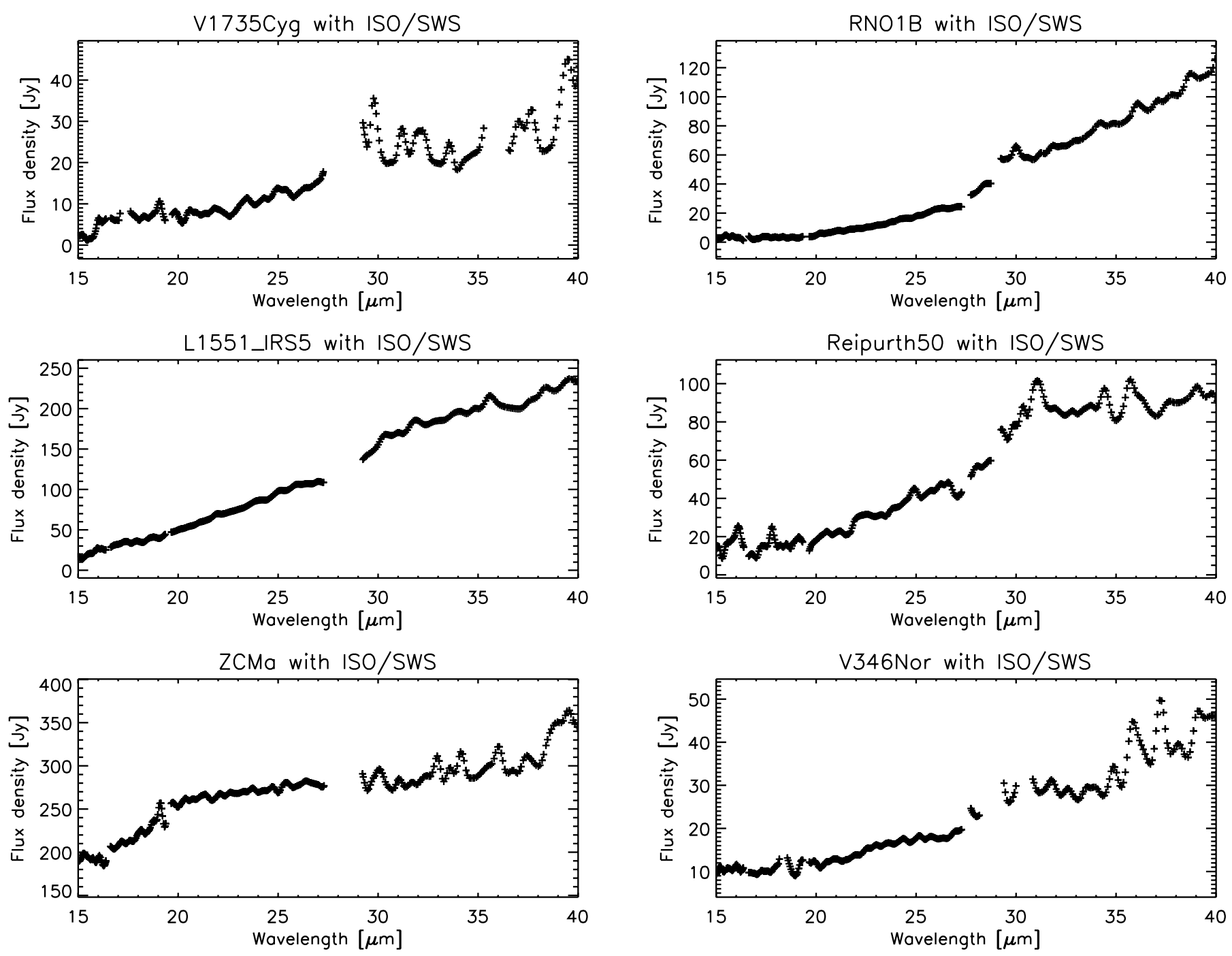

Fig. 3.-Same as Fig. 1, but between 15 and $40 \mu \mathrm{m}$. All spikes seen in the data are artifacts and not believed to be real, as they showed up either in the up or in the down scan but not in both.

on ground-based observations, but a firm confirmation was thus far lacking. We discuss the spectrum of Parsamian 21 in more detail below.

For a better comparison of the emission features, we plotted in Figure 11 the normalized fluxes of the objects between 8 and $13 \mu \mathrm{m}$. Following van Boekel et al. (2005), the normalization was done via

$$
F_{\text {norm }}(\lambda)=1+\frac{F_{\text {obs }}(\lambda)-F_{\text {cont }}(\lambda)}{\left\langle F_{\text {cont }}\right\rangle},
$$

where $F_{\text {obs }}(\lambda)$ is the observed flux, $F_{\text {cont }}(\lambda)$ is the continuum flux, and $\left\langle F_{\text {cont }}\right\rangle$ denotes the mean value of the underlying continuum in the considered wavelength regime. This normalization ensures that the shape of the emission feature is preserved. From Figure 11 it becomes clear that the emission features of Bran 76, FU Ori, and V1515 Cyg are quite similar in terms of shape and strength. The features of V1057 Cyg and XZ Tau are far less pronounced and much broader. ${ }^{8}$ The emission profiles of V1647 Ori and Parsamian 21 are slightly stronger than those of the other ob-

\footnotetext{
8 In contrast to our analysis, Green et al. (2006) found the feature of V1057 Cyg to be comparable in shape (and thus in dust composition) to those of Bran 76, FU Ori, and V1515 Cyg.
}

jects, and, as mentioned above, the latter object is the only one showing strong PAH emission bands. The three epochs of data for V1647 Ori allow us to study the variability of this object in the $10 \mu \mathrm{m}$ region over a period of approximately 5 months. Figure 11 shows that between 2004 October (epoch 1) and the middle of March 2005 (epoch 2) the flux level decreased significantly. At the third epoch (end of March 2005) the flux appears to have slightly increased again (see also Fig. 5). The overall shape of the feature during the 6 month period, however, did not change.

To put these results in a broader context with other young objects and to get a first idea on the dust grain properties, we plot in Figure 12 the flux ratio at 11.3 and $9.8 \mu \mathrm{m}$ against the computed peak over the continuum in the normalized spectra, i.e., the maxima of equation (1). This figure also shows the region typically occupied by young $\mathrm{T}$ Tauri stars and the slightly more massive HAeBes (see, e.g., Przygodda et al. 2003; van Boekel et al. 2003, 2005; Kessler-Silacci et al. 2006). We find that the objects presented here tend to have in general a relatively weak peak over continuum emission and a flux ratio between 0.8 and 1.0. While the strength of the peak over the continuum is interpreted as a tracer for grain sizes (with higher peak values denoting smaller grains), the flux ratio is a more general tracer for grain processing, i.e., grain growth as well as crystallization. This is explained 

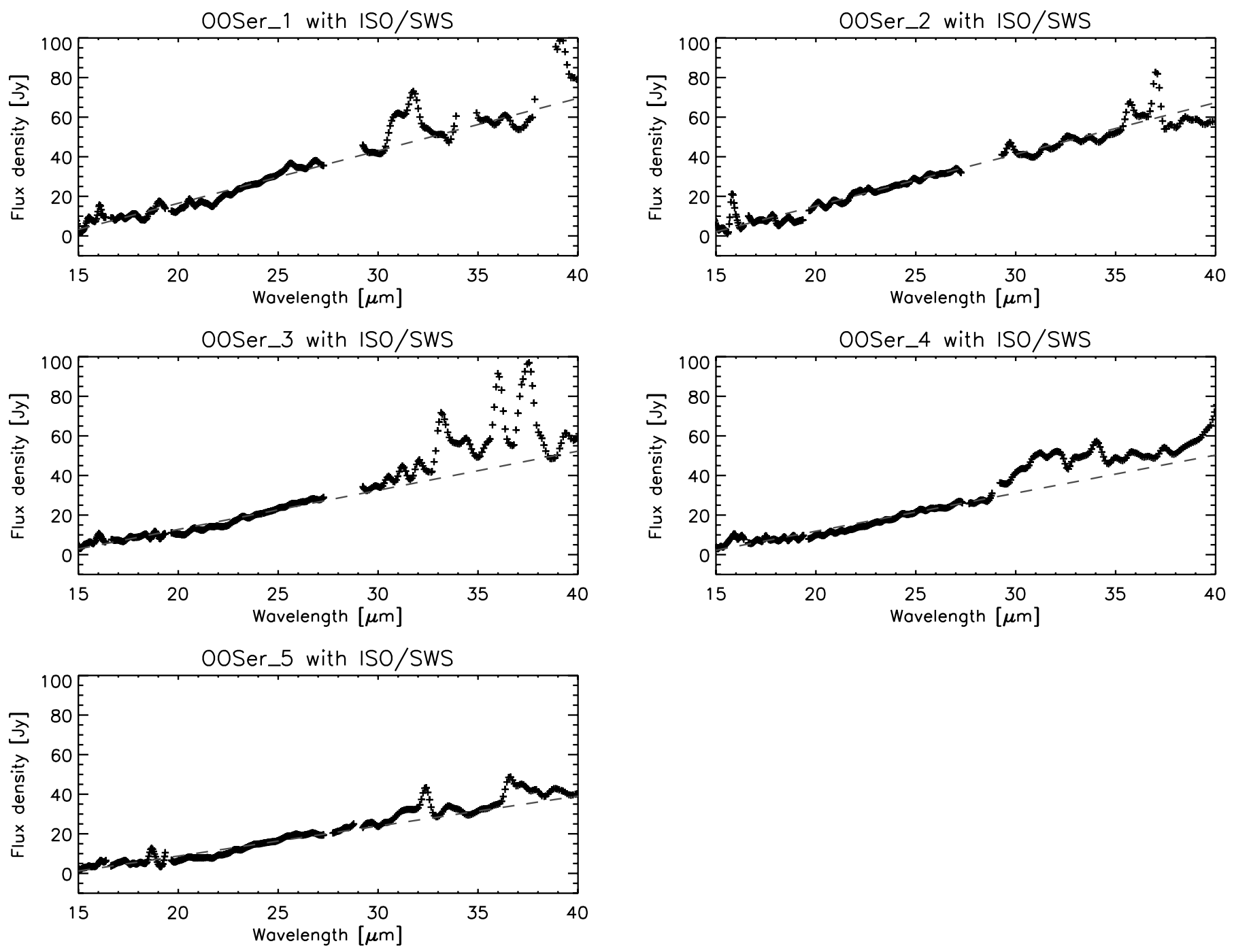

FIG. 4.- Same as Fig. 2, but between 15 and $40 \mu \mathrm{m}$. The dashed line illustrates a fit to the data between 15 and $30 \mu \mathrm{m}$ to estimate the change of the slope and flux

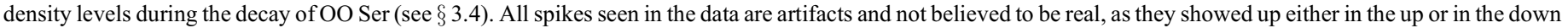
scan but not in both. [See the electronic edition of the Journal for a color version of this figure.]

by the fact that not only the growing of grains leads to a broader and flatter silicate feature between 9.8 and $12.0 \mu \mathrm{m}$ (Bouwman et al. 2001), but also the crystallization process introduces distinct emission peaks of forsterite and enstatite longward of $10 \mu \mathrm{m}$ (see, e.g., van Boekel et al. 2005). Taking these considerations into account, we find that our sample shows clear evidence for grain growth and dust processing. For a more quantitative analysis of the dust composition we fitted a dust model to the data as explained in the following subsection.

\subsubsection{Dust Composition}

In Figure 13 we fitted an analytical dust model to the spectrum of FU Ori between 7 and $17 \mu \mathrm{m}$. To equally weight the low- and high-resolution part of the spectrum, we rebinned the data to a spectral resolution of 100 , still sufficient to disentangle contributions from different dust species. Instead of fitting a two-component model to the data, with one temperature for the underlying continuum and one temperature for the emission layer, as we did in Quanz et al. (2006), we use a new model described in A. Juhász et al. (2007, in preparation). In this model the temperatures for the disk midplane, the disk surface layer, and an inner disk rim are fitted by power-law distributions instead of single blackbody temperatures. In this respect, the model represents more realistically the temperature distribution in a protoplanetary disk. The input parameters for the model were taken from the simple accretion disk model in Quanz et al. (2006). Table 4 summarizes the fitted parameters of the individual temperature components.

The dust model was based on opacities for six dust species (amorphous olivine and pyroxene, crystalline forsterite and enstatite, silica, and amorphous carbon) in three grain sizes $(0.1,1.5$, and $6.0 \mu \mathrm{m})$. References for the optical properties of the grains are given in A. Juhász et al. (2007, in preparation) and Quanz et al. (2006). In contrast to the analysis presented in Quanz et al. (2006), this time we included also carbon grains in the fit. However, as shown in Figure 13 and summarized in Table 5, the best fit $\left(\chi^{2}=45.3\right)$ does not require any carbon grains. The derived mass fractions agree well with the results based on the more simple twocomponent dust model used in Quanz et al. (2006). While a large fraction of big, amorphous grains contribute to a high-temperature continuum, the smaller grains are responsible for the observed emission feature. Still, we emphasize that, according to our analysis, also in the disk surface layer large grains are present: comparing the derived composition to that of the ISM (Kemper et al. 

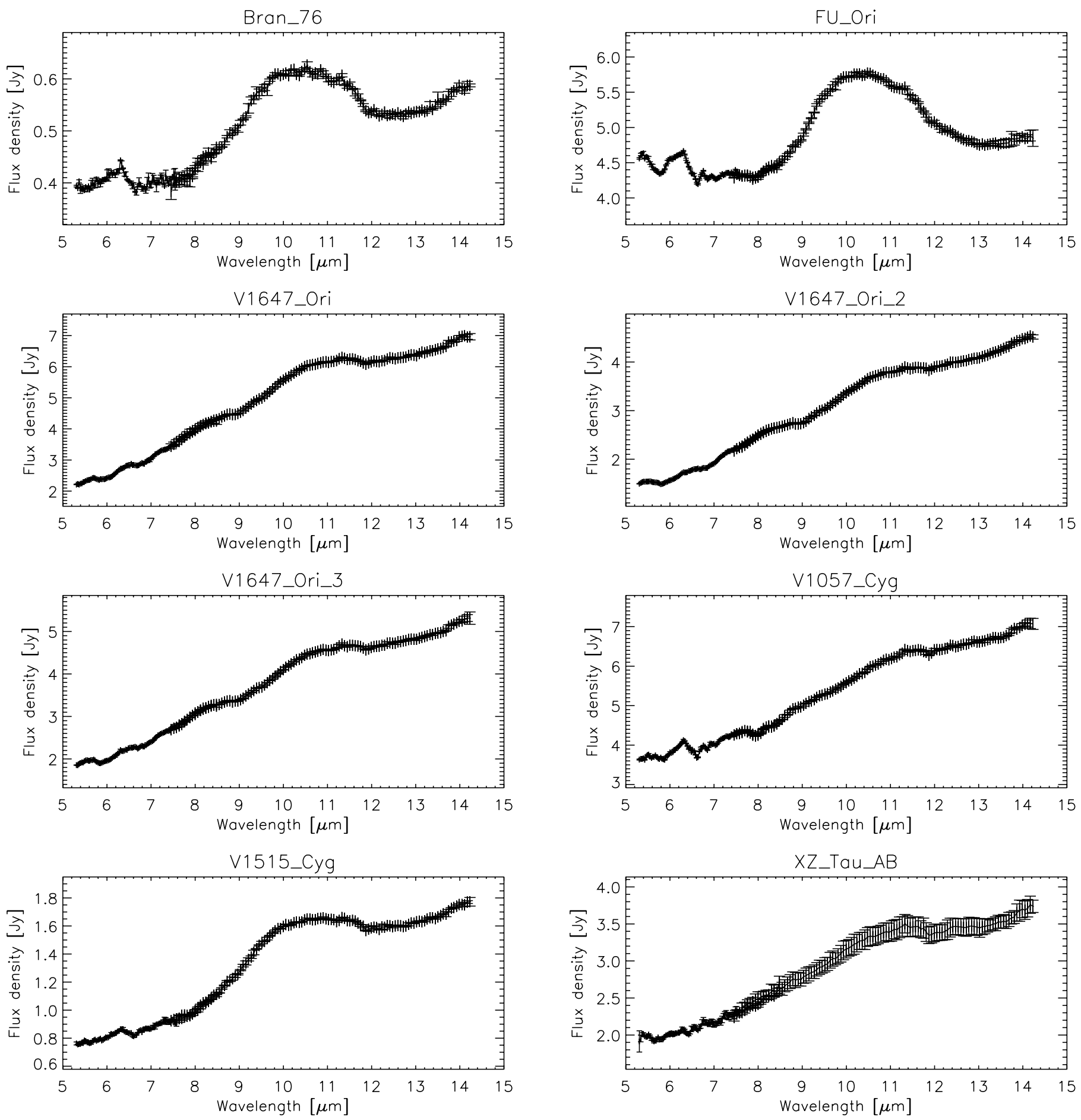

FIG. 5.-Spitzer low-resolution spectra between 5 and $14 \mu \mathrm{m}$.

2004; van Boekel et al. 2005), we find a significantly higher fraction of $1.5 \mu \mathrm{m}$ grains in the dust of FU Ori. Furthermore, the model does predict a negligible amount of crystalline grains $(<0.01)$, no silica dust, and, as mentioned above, no carbon grains. Certainly, the values for the derived mass fractions should not be taken literally, as, for instance, the temperature structure in the disk and the dust opacities are not perfectly known. However, the models presented here and in Quanz et al. (2006) agree on two very important aspects: (1) there is clear evidence for dust coagulation in the spectra of FUors, and (2) there is hardly any contribution from crystalline grains detectable in the data. This lack of crystal- line grains was already noticed and discussed in Quanz et al. (2006), but we reinvestigate this issue below.

\subsubsection{The PAH Features of Parsamian 21}

As its features are significantly different than those of the other FUors, we plot in Figure 14 the dereddened spectrum of Parsamian 21 and analyze it in more detail. The vertical lines illustrate where typically PAH emission bands are located. While prominent PAH emission is present at 6.3, 8.2, and (possibly) $11.3 \mu \mathrm{m}$, no bands are seen at 7.7, 8.6, and $12.7 \mu \mathrm{m}$. In addition, the spectrum bears signs of underlying silicate emission and it is 

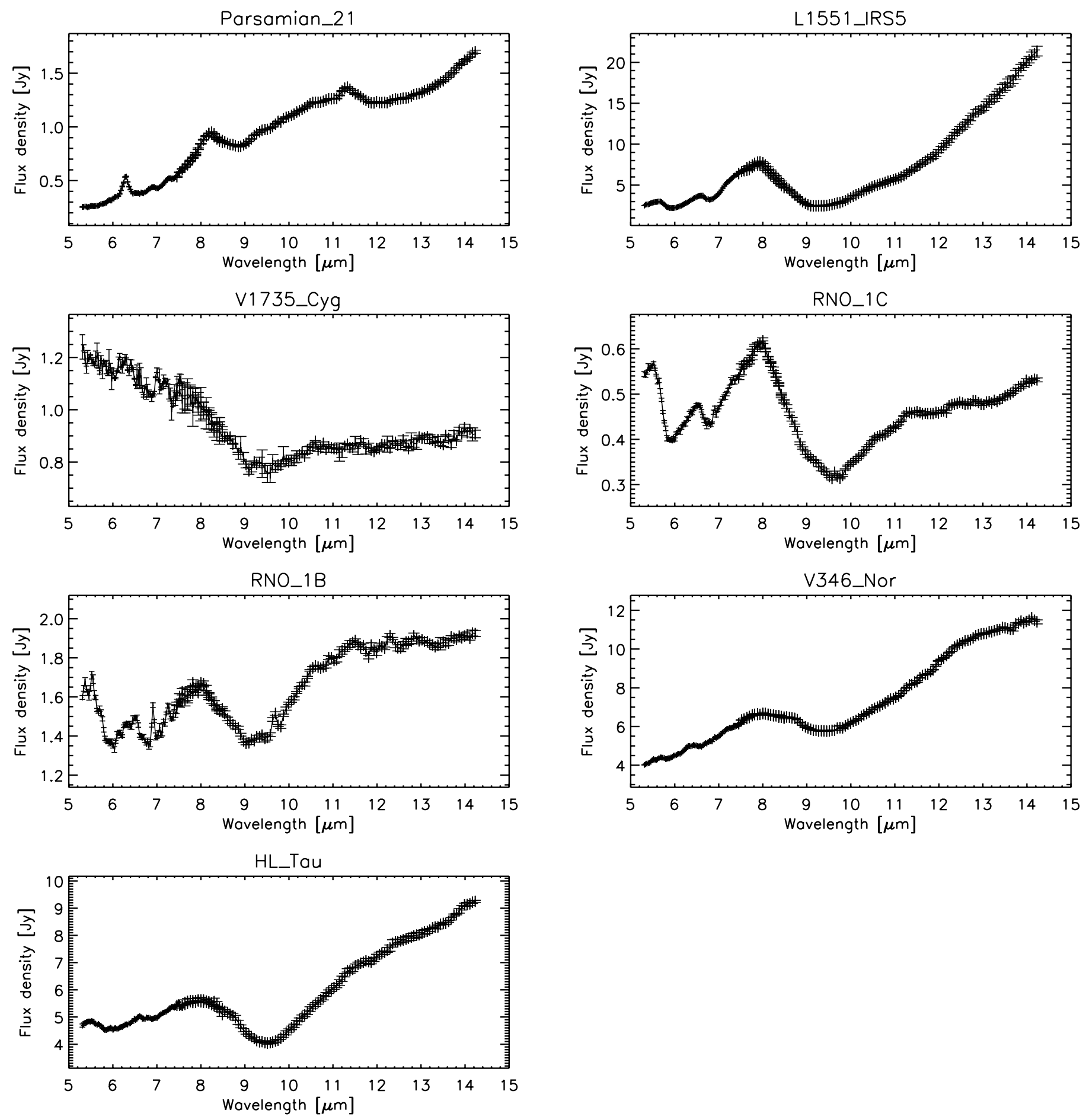

FIG. 6.-Same as Fig. 5, but for different objects.

difficult to disentangle in the $11.3 \mu \mathrm{m}$ emission band the possible contributions from crystalline forsterite and PAHs.

PAHs are commonly seen in the spectra of HAeBes (e.g., Meeus et al. 2001; Acke \& van den Ancker 2004; Sloan et al. 2005), and more recently, PAH emission was also detected in T Tauri spectra (Geers et al. 2006). However, the spectrum of Parsamian 21 differs significantly from the PAH spectra of other young objects: it shows a broad and strong feature at $8.2 \mu \mathrm{m}$ that is barely detected and the main characteristic of group C objects in Peeters et al. (2002). These objects show no PAH complex at 7.7 and $8.6 \mu \mathrm{m}$ but the peculiar broad band at $8.2 \mu \mathrm{m}$ whose origin remains yet unknown (Peeters et al. 2002). In $\S 4.3$ we critically review the findings presented here and discuss their implications with respect to the FUor status of Parsamian 21.

\subsubsection{Qualitative Analyses of the 5-8 $\mu \mathrm{m}$ Region}

As already noted and explained by Green et al. (2006), FU Ori, Bran 76, V1515 Cyg, and also V1057 Cyg show absorption bands of gaseous $\mathrm{H}_{2} \mathrm{O}$ at 5.8 and $6.8 \mu \mathrm{m}$ (Fig. 5) coming from 

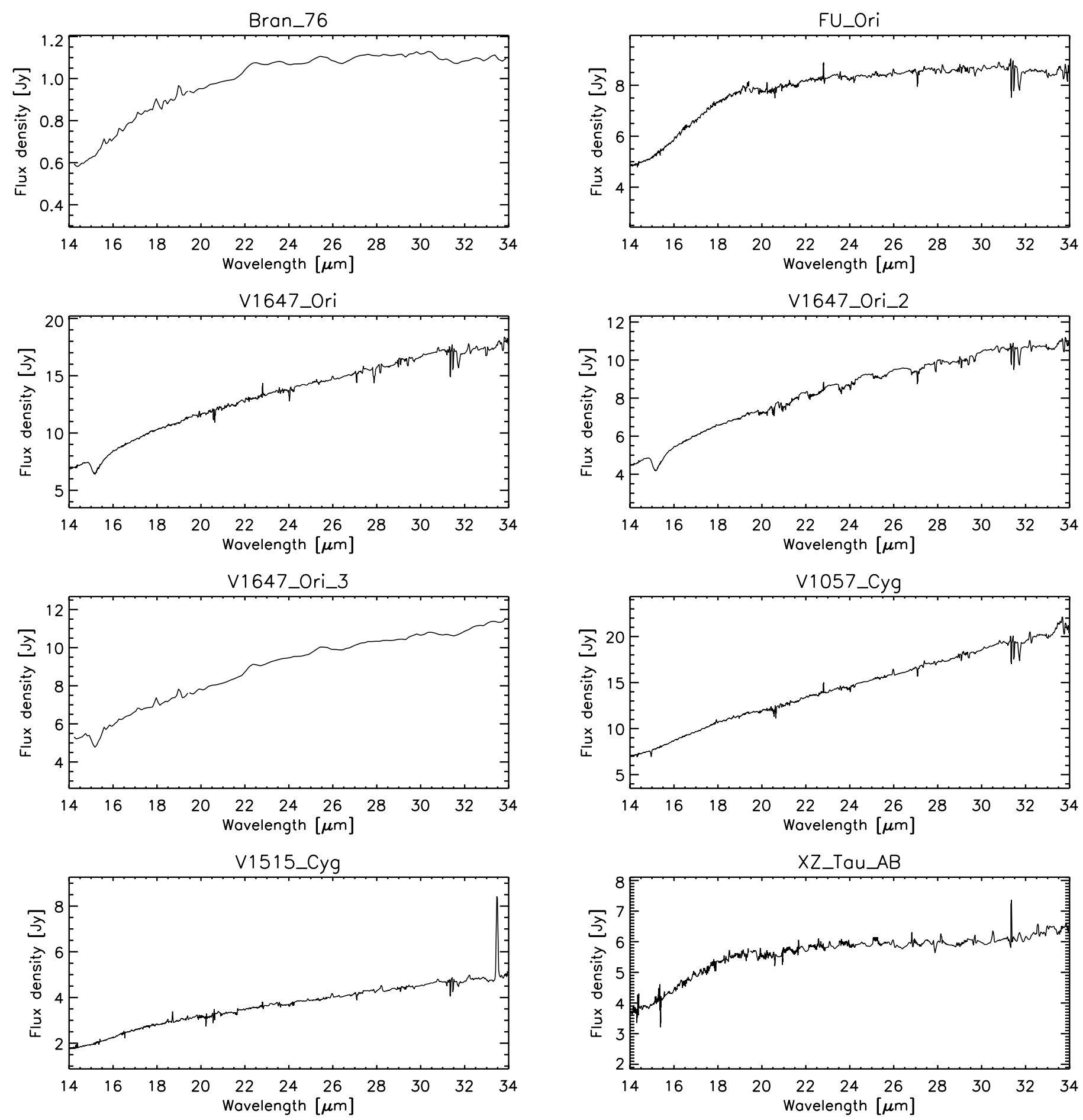

FIG. 7.-Same objects as in Fig. 5, but now showing the high-resolution part of the spectrum between 14 and $34 \mu \mathrm{m}$. The spectrum of V1515 Cyg shows a prominent emission line longward of $33 \mu \mathrm{m}$, which is discussed in $\S 3.5$. Between 31 and $32 \mu \mathrm{m}$ all objects where high-resolution data are present show artifacts from the data reduction process.

a collection of rotation-vibration bands. ${ }^{9}$ V1647 Ori, however, shows evidence for ice absorption bands at 6.0 and $6.85 \mu \mathrm{m}$ (see also Fig. 20 below). We mention already here that this object shows an additional ice feature at $15.2 \mu \mathrm{m}$ due to $\mathrm{CO}_{2}$ (see Fig. 19 below). V1647 Ori is the only object in the sample showing the $10 \mu \mathrm{m}$ silicate feature in emission accompanied by sig-

\footnotetext{
9 Interestingly, already Sato et al. (1992) found evidence for water vapor absorption between 1 and $3 \mu \mathrm{m}$ in the spectra of FU Ori, V1515 Cyg, and V1057 Cyg, but also L1551 IRS 5, V1057 Cyg, and Z CMa.
}

nificant ice absorption bands. A more detailed analysis of these ice features is given in $\S 3$ 3.3. Finally, as we discuss in the following section, Parsamian 21 shows clear evidence for a PAH emission feature at $6.2 \mu \mathrm{m}$.

\subsection{Objects with $10 \mu \mathrm{m}$ Absorption}

\subsubsection{Analyses of the $10 \mu \mathrm{m}$ Region}

In Figure 15 we plot the optical depth in the $10 \mu \mathrm{m}$ region for the objects showing the silicate band in absorption. To derive the 

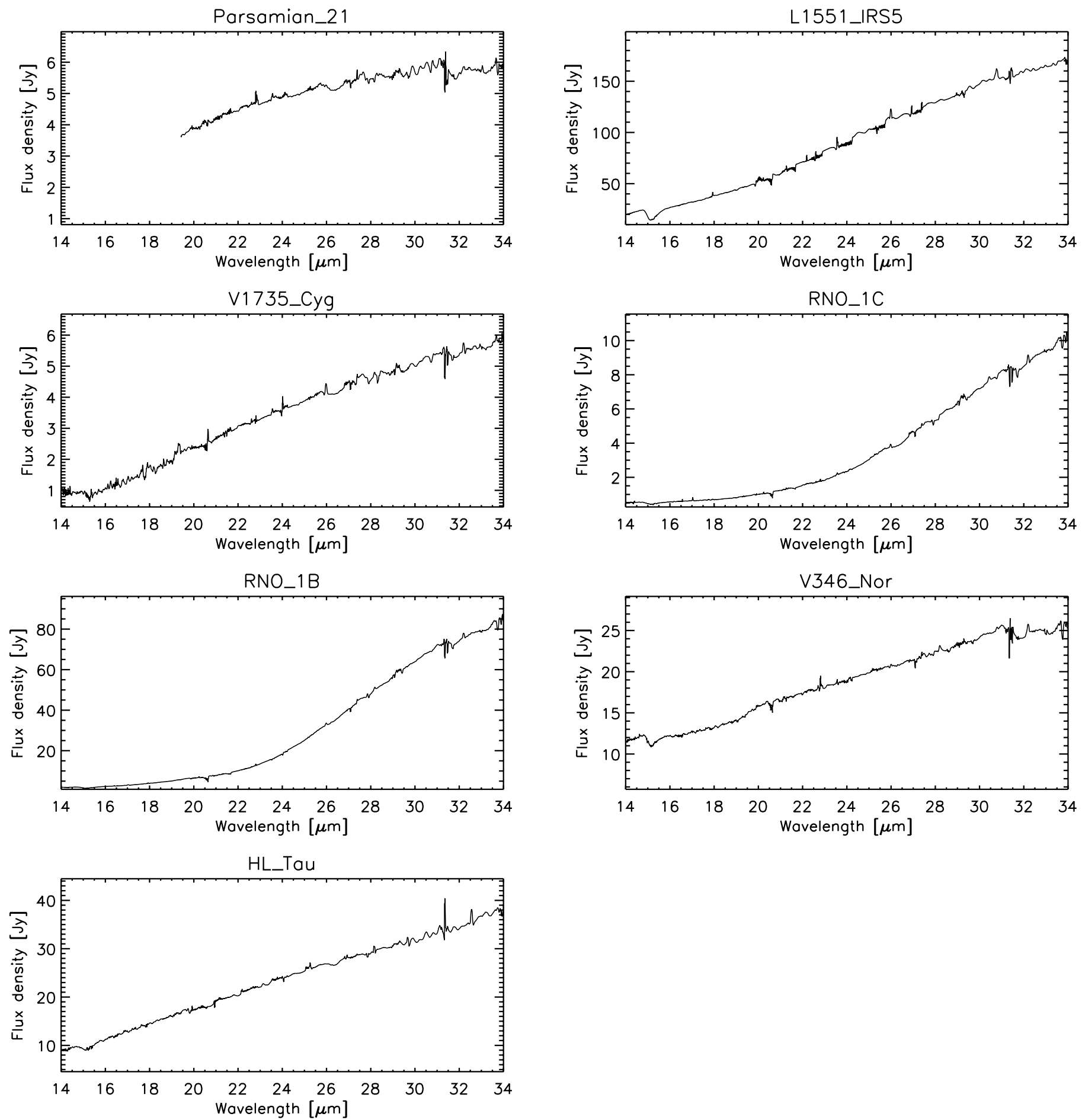

Fig. 8. - Same objects as in Fig. 6, but now showing the high-resolution part of the spectrum between 14 and $34 \mu \mathrm{m}$. Between 31 and $32 \mu \mathrm{m}$ all objects where highresolution data are present show artifacts from the data reduction process.

optical depth, we fitted the continuum with a polynomial of first order to the observed spectra between 8 and $13 \mu \mathrm{m}$, assuming that no absorption due to silicates is present at these wavelengths. Also, here the exact shape of the continuum is difficult to estimate and any fit always carries uncertainties. However, even fits with higher order polynomials did not alter the results significantly, and the main conclusions remained valid. Based on the assumed continuum, we then computed the optical depth $\tau$ using

$$
F_{\text {obs }}(\lambda)=F_{\text {cont }}(\lambda) e^{-\tau}
$$

where $F_{\text {obs }}(\lambda)$ is the observed flux and $F_{\text {cont }}(\lambda)$ is the assumed continuum. To derive the wavelength position and value of the maximum optical depth, we did the following: As some spectra are quite noisy (e.g., V1735 Cyg, RNO 1B, Z CMa, and Reipurth 50), we fitted the data between 8.8 and $10.2 \mu \mathrm{m}$, i.e., the region with the greatest optical depth, with a polynomial of second order (shown as dot-dashed lines in Fig. 15). The wavelength position of the maximum optical depth (vertical dot-dashed lines in Fig. 15) and the corresponding value are then derived from the fit. From the spectra with a high signal-to-noise ratio and strong 
L1551 IRS 5

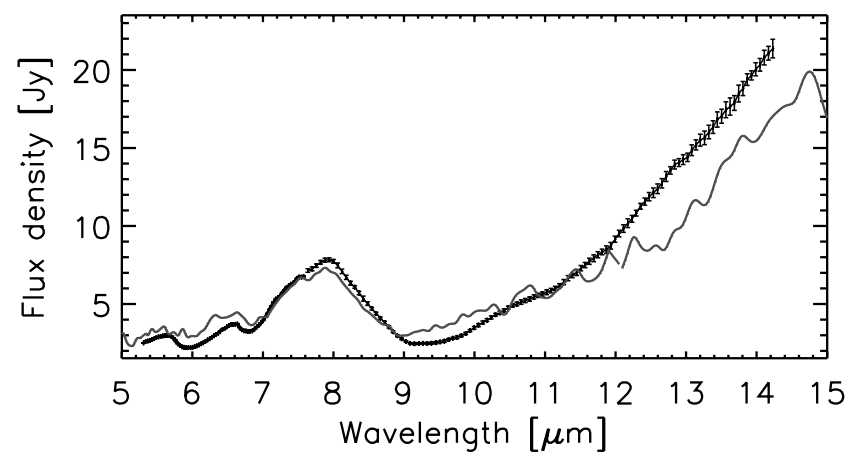

RNO 1B

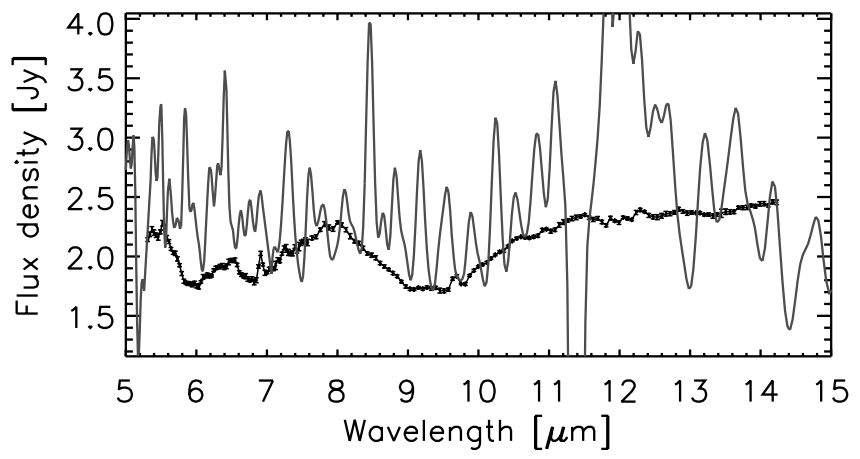

V346 Nor

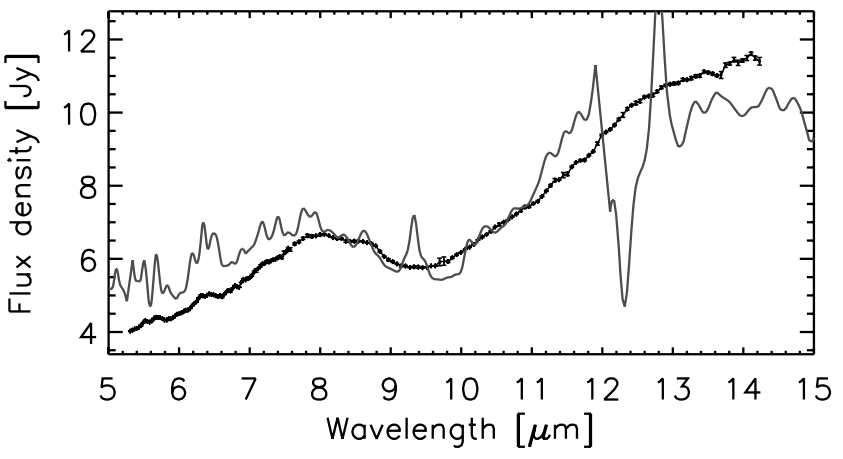

V1735 Cyg

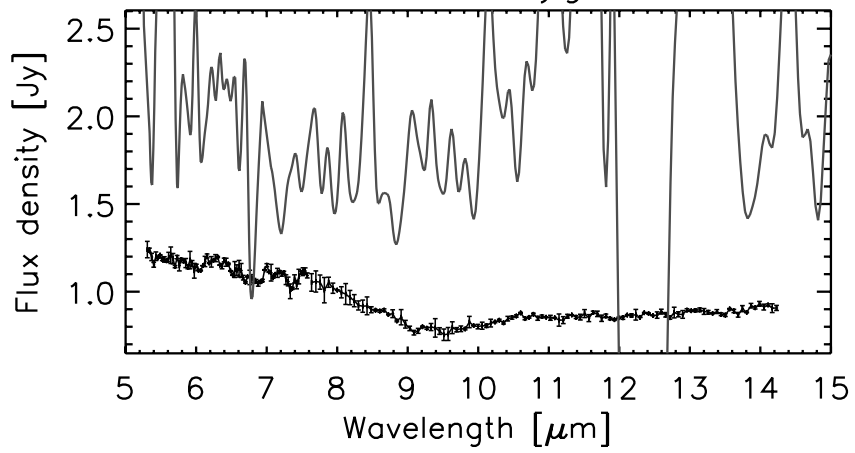

FIG. 9.-Comparison between ISO SWS and Spitzer IRS data for objects observed with both satellites. The ISO data (solid lines) are significantly more noisy. In our sample only data for objects with high flux levels or long integration times are suitable for quantitative analyses. [See the electronic edition of the Journal for a color version of this figure.]

TABLE 3

Overview of Prominent Spectroscopic Features (Mostly Ices and Dust) Seen in the Spectra Presented in Figures 1, 2 , 5, 6, 7, and 8

\begin{tabular}{|c|c|c|c|c|c|c|c|c|}
\hline Object & Instrument & $\begin{array}{c}\mathrm{H}_{2} \mathrm{O}(\text { Ice }) \\
3.08 \mu \mathrm{m}\end{array}$ & $\begin{array}{c}\mathrm{H}_{2} \mathrm{CO}(?) / \mathrm{HCOOH}(?) / \mathrm{H}_{2} \mathrm{O}(\text { Ice }) \\
5.85 \mu \mathrm{m} / 6.0 \mu \mathrm{m}\end{array}$ & $\begin{array}{c}\mathrm{H}_{2} \mathrm{O} \text { (Gas) } \\
5.8 \mu \mathrm{m} / 6.8 \mu \mathrm{m}\end{array}$ & $\begin{array}{c}\mathrm{CH}_{3} \mathrm{OH}(?) / \mathrm{NH}_{4}^{+}(?) \\
6.85 \mu \mathrm{m}\end{array}$ & $\begin{array}{l}\text { Silicates } \\
10.0 \mu \mathrm{m}\end{array}$ & $\begin{array}{c}\mathrm{CO}_{2}(\text { Ice }) \\
15.2 \mu \mathrm{m}\end{array}$ & $\mathrm{PAH}^{\mathrm{a}}$ \\
\hline 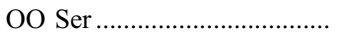 & ISO SWS & $\ldots$ & $\ldots$ & $\ldots$ & $\ldots$ & abs & abs & $\ldots$ \\
\hline \multirow[t]{2}{*}{ 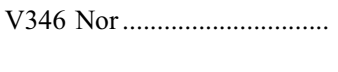 } & ISO SWS & abs & abs & $\ldots$ & $\ldots$ & abs & $\ldots$ & $\ldots$ \\
\hline & Spitzer IRS & $\ldots$ & abs & $\ldots$ & abs & abs & abs & $\ldots$ \\
\hline $\mathrm{Z} \mathrm{CMa}$ & ISO SWS & $\ldots$ & $\ldots$ & $\ldots$ & $\ldots$ & abs & $\ldots$ & $\ldots$ \\
\hline 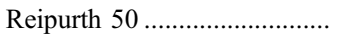 & ISO SWS & abs & abs & $\ldots$ & abs & abs & $\ldots$ & $\ldots$ \\
\hline \multirow[t]{2}{*}{ 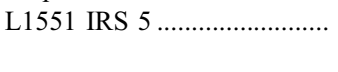 } & ISO SWS & abs & abs & $\ldots$ & abs & abs & abs & $\ldots$ \\
\hline & Spitzer IRS & $\ldots$ & abs & $\ldots$ & abs & abs & abs & $\ldots$ \\
\hline 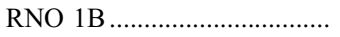 & ISO SWS & abs & $\ldots$ & $\ldots$ & $\ldots$ & flat & $\ldots$ & $\ldots$ \\
\hline 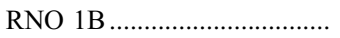 & Spitzer IRS & $\ldots$ & abs & $\ldots$ & abs & abs & abs & $\ldots$ \\
\hline 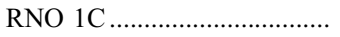 & Spitzer IRS & $\ldots$ & abs & $\ldots$ & abs & abs & abs & $\ldots$ \\
\hline \multirow[t]{2}{*}{ 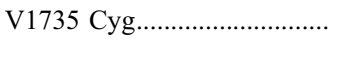 } & ISO SWS & abs & abs? & $\ldots$ & $\ldots$ & flat & $\ldots$ & $\ldots$ \\
\hline & Spitzer IRS & $\ldots$ & $\ldots$ & $\ldots$ & $\ldots$ & abs & abs? & $\ldots$ \\
\hline 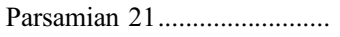 & Spitzer IRS & $\ldots$ & $\ldots$ & $\ldots$ & $\ldots$ & $\mathrm{em}$ & $\ldots$ & em \\
\hline Bran 76 (BBW 76)............... & Spitzer IRS & $\ldots$ & $\ldots$ & abs & $\ldots$ & em & $\ldots$ & $\ldots$ \\
\hline 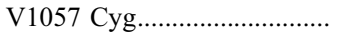 & Spitzer IRS & $\ldots$ & $\ldots$ & abs & $\ldots$ & em & abs (gas) & $\ldots$ \\
\hline 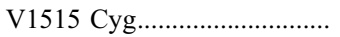 & Spitzer IRS & $\ldots$ & $\ldots$ & abs & $\ldots$ & em & $\ldots$ & $\ldots$ \\
\hline 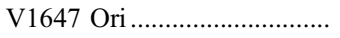 & Spitzer IRS & $\ldots$ & abs & $\ldots$ & abs & em & abs & $\ldots$ \\
\hline 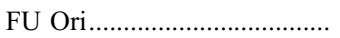 & Spitzer IRS & $\ldots$ & $\ldots$ & abs & $\ldots$ & em & $\ldots$ & $\ldots$ \\
\hline 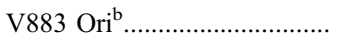 & ESO Timmi2 & $\ldots$ & $\ldots$ & $\ldots$ & $\ldots$ & abs & $\ldots$ & $\ldots$ \\
\hline 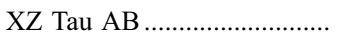 & Spitzer IRS & $\ldots$ & $\ldots$ & $\ldots$ & $\ldots$ & em & $\ldots$ & $\ldots$ \\
\hline HL Tau ................................. & Spitzer IRS & $\ldots$ & abs & $\ldots$ & abs & abs & abs & $\ldots$ \\
\hline
\end{tabular}

Notes.-Here "abs" denotes an absorption feature, “em” an emission feature. For objects where ISO and Spitzer data are available, the Spitzer data are of higher quality and thus more reliable.

a Any significant emission at 6.2 or $7.7 \mu \mathrm{m}(\mathrm{C}-\mathrm{H}$ modes $)$, at $8.6,11.3$, or $12.7 \mu \mathrm{m}(\mathrm{C}-\mathrm{C}$ modes $)$, or at $8.2 \mu \mathrm{m}($ origin not clear yet).

b Observations from Schütz et al. (2005). 

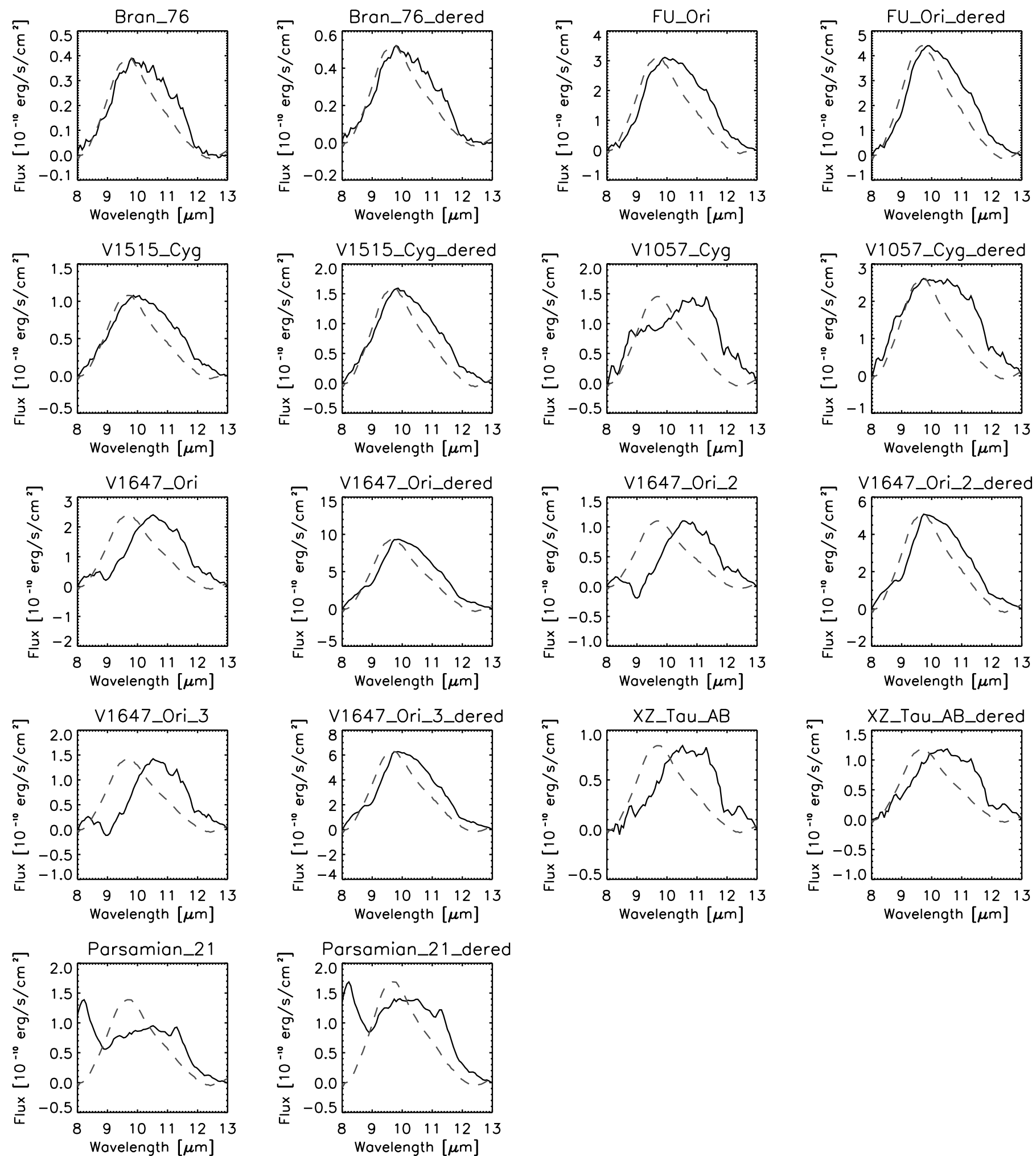

FIG. 10.-Continuum-subtracted silicate emission profiles (solid lines). Each spectrum is shown twice, i.e., with and without being corrected for interstellar extinction as explained in the text. The assumed values for $A_{V}$ are $2.2 \mathrm{mag}$ (Bran 76; Reipurth et al. 2002), 2.4 mag (FU Ori; Skinner et al. 2006 ), 3.2 mag (V1515 Cyg; Herbig 1977), 3.2 mag (V1057 Cyg; Herbig 1977), 11.0 mag (V1647 Ori; Muzerolle et al. 2005), 1.4 mag (XZ Tau; White \& Ghez 2001), and 4.0 mag (Parsamian 21; Staude $\&$ Neckel 1992). We used the extinction law from Mathis (1990) with $R_{V}=3.1$. For comparison the dashed line shows the typical ISM silicate feature observed toward

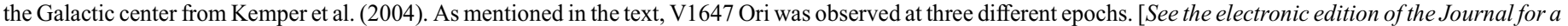
color version of this figure.] 

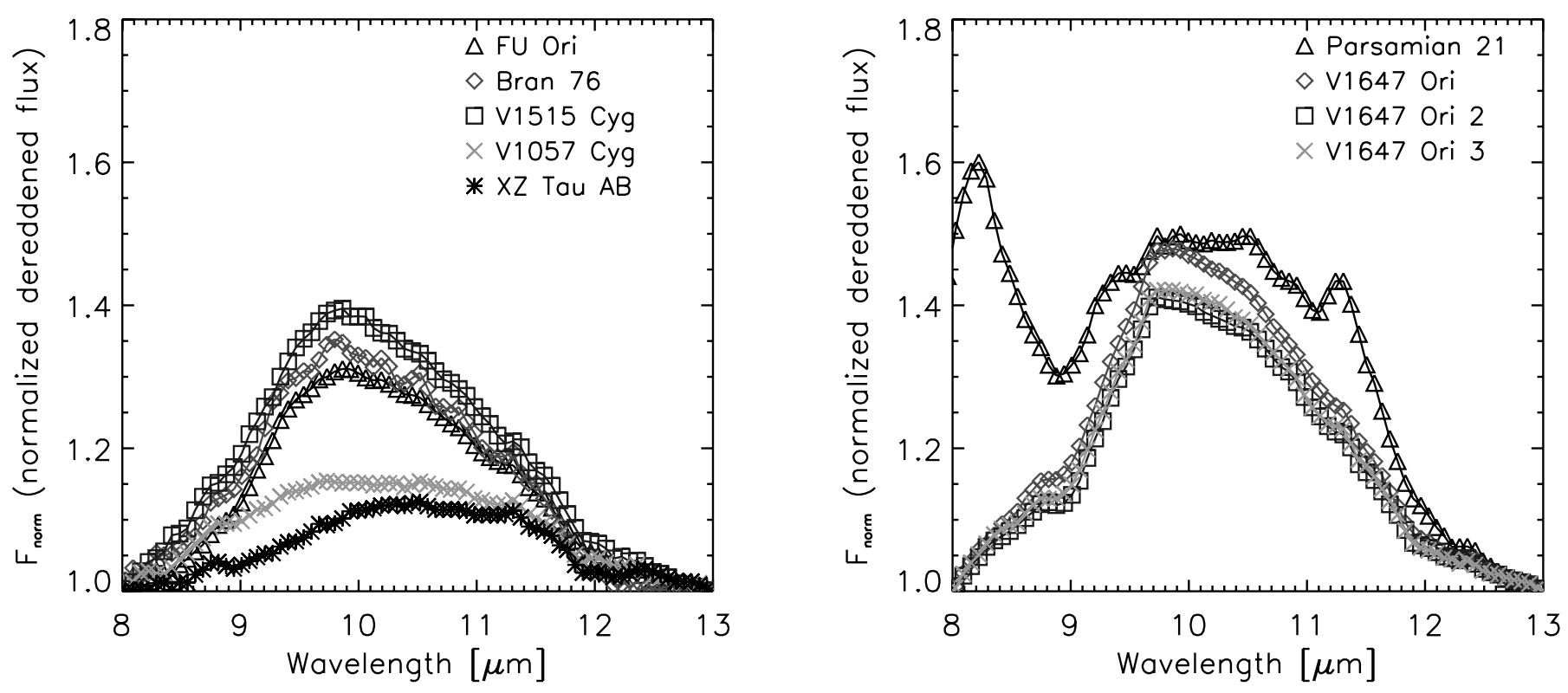

Fig. 11.-Dereddened and normalized $10 \mu \mathrm{m}$ dust emission features of the objects shown in Fig. 10. For a better comparison the dereddened spectra are shown. [See the electronic edition of the Journal for a color version of this figure.]

absorption profiles (e.g., the Spitzer spectra of L1551 IRS 5 and HL Tau) it becomes clear that the fit matches the shape of the absorption feature quite well and that this approach seems reasonable. The dotted horizontal line in Figure 15 shows the assumed continuum level. In Table 6 we summarize the derived maximum optical depth for each object $\tau_{\text {silicate }}$ and the corresponding wavelength. We give also values for the extinction in the silicate band $A_{\text {silicate }}$ and estimates for the corresponding extinction in the optical $A_{V}, A_{\text {silicate }}$ was derived via

$$
A_{\text {silicate }}=-2.5 \log _{10}\left(e^{-\tau_{\text {silicate }}}\right)
$$

The errors for $\tau_{\text {silicate }}$ and $A_{\text {silicate }}$ are based on the $1 \sigma$ uncertainties in the spectra and the resulting fluctuations of the poly-

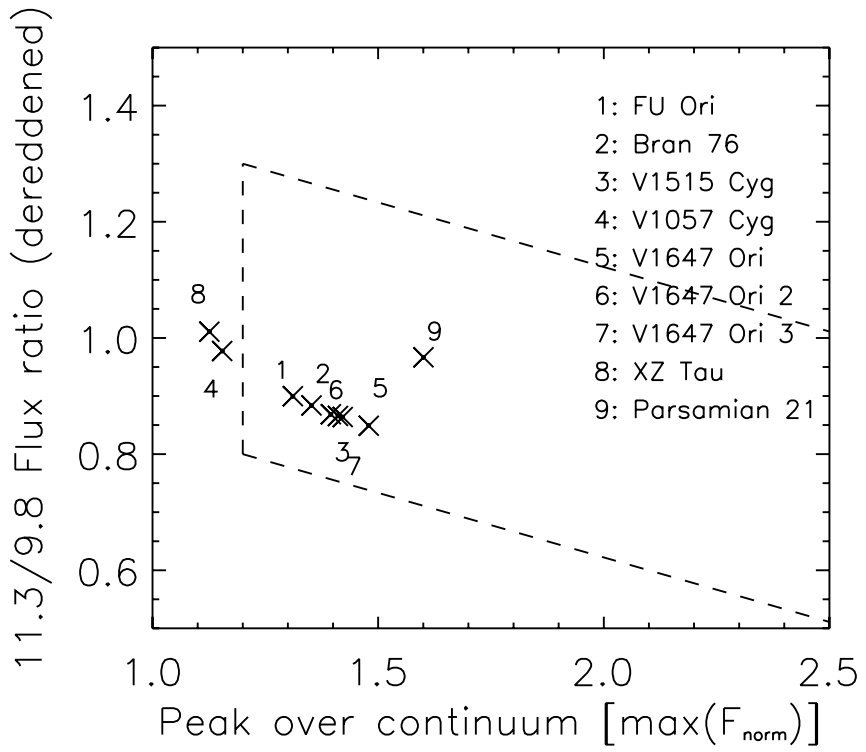

FIG. 12.-Flux ratio between 11.3 and $9.8 \mu \mathrm{m}$ plotted against the normalized peak over the continuum. The dashed line indicates the region where typically $\mathrm{T}$ Tauri stars (Przygodda et al. 2003) and HAeBes (van Boekel et al. 2003, 2005) are located. nomial fit. $A_{V}$ and its error are derived from averaging over the extinction models of Mathis (1990) and Weingartner \& Draine (2001) and assuming two different values of $R_{V}$ for each model $\left(R_{V}=3.1\right.$ and 5.0 for Mathis $1990 ; R_{V}=3.0$ and 5.5 for Weingartner \& Draine 2001). As already noted by Cohen \& Kuhi (1979), one has to be careful when deriving a value for the optical extinction based on the silicate feature, as uncertainties in the underlying continuum and unknown dust compositions influence the results. Hence, for comparison, Table 6 gives also

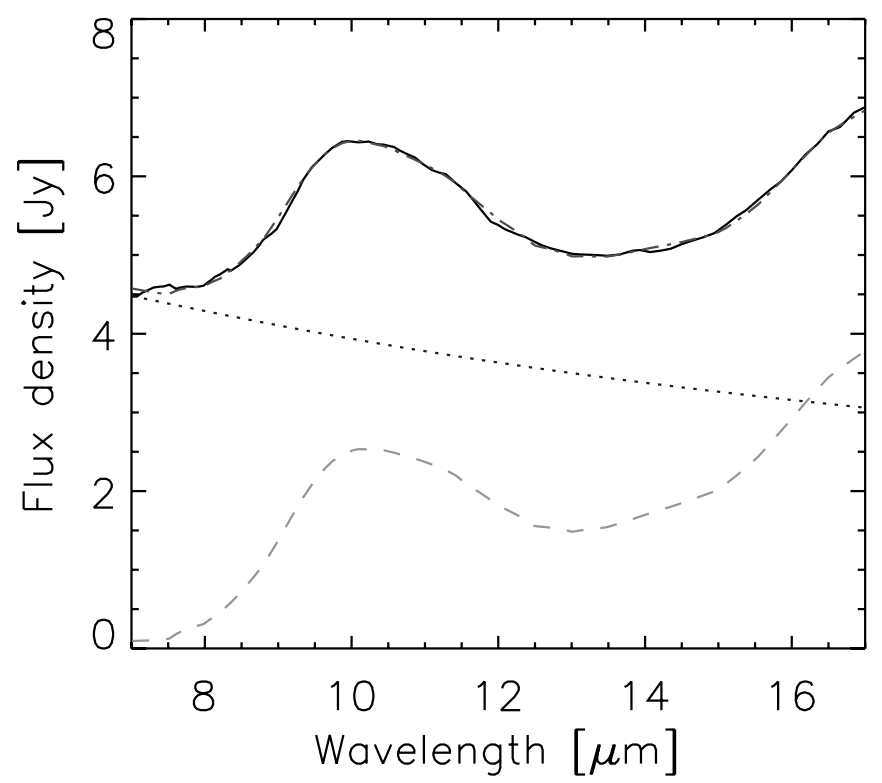

FIG. 13.- Results from a dust model fit to the $7-17 \mu \mathrm{m}$ region of the spectrum of FU Ori using the model described in the text. The observed spectrum is shown as the solid line, the dotted line illustrates the contribution from the fitted continuum, and the dashed line denotes the computed emission feature. The sum of the fitted components is shown as the dot-dashed line, matching the observed spectrum. The mass fractions of the fitted dust species are given in Table 5. The spectrum is fitted solely with amorphous grains, part of which have grown significantly. [See the electronic edition of the Journal for a color version of this figure.] 
TABLE 4

Temperatures and Power-Law Indices for the Different Components of the Analytical Dust Model for FU Ori Shown in Figure 13

\begin{tabular}{|c|c|c|}
\hline Component & $\begin{array}{c}T \\
(\mathrm{~K})\end{array}$ & Exponent $p$ \\
\hline 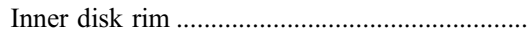 & 2246 & -0.84 \\
\hline 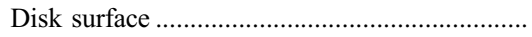 & 1128 & -0.49 \\
\hline 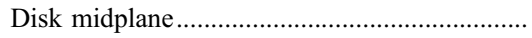 & 865 & -0.11 \\
\hline
\end{tabular}

values for $A_{V}$ found in the literature. One has to keep in mind, however, that also here certain assumptions have been made and partly different observing techniques were applied, potentially leading to discrepancies in the derived values for $A_{V}$.

For V346 Nor and RNO $1 \mathrm{C}$ the values for $A_{V}$ agree quite well. Also for Z CMa, Reipurth 50, and L1551 IRS 5 the values are in general agreement. Due to the high extinction toward the latter two objects, a good estimate of $A_{V}$ is difficult. We would like to point out, however, that a value of $A_{V} \gtrsim 150 \mathrm{mag}$ for L1551 IRS 5 as found by Campbell et al. (1988) and mentioned, for instance, by Stocke et al. (1988), White et al. (2000), and Fridlund et al. (2002) appears to be an overestimate. In yet unpublished NIR data taken with the Omega2000 camera at the Calar Alto Observatory (Spain) we do see an NIR counterpart to the L1551 IRS 5 system with $K_{S} \approx 9.7 \mathrm{mag}$. Even if this was purely scattered light from a disk, the intrinsic $K$ magnitude of the central object(s) (it might be a multiple system) cannot be lower. Thus, the observed $K$ magnitude is a lower limit for the intrinsic $K$ luminosity of the source. The above-mentioned value for $A_{V}$ would translate into $A_{K} \approx 15 \mathrm{mag}$, and thus the absolute $K$-band magnitude of L1551 IRS 5 would be approximately -11 mag for an assumed distance of $140 \mathrm{pc}$. For young low-mass objects this appears to be definitely too luminous even if accretion luminosities are taken into account as well. For V1735 Cyg and HL Tau our derived values for $A_{V}$ are lower than those found in the literature. However, for V1735 Cyg no errors are given for the value found in the literature. For HL Tau the optical extinction derived from the radiative transfer model by Men'shchikov et al. (1999) may suffer from insufficient information about the source geometry or dust opacities, which can easily lead to large uncertainties in the computed figures. The comparison for these objects clearly shows that, in particular for embedded objects, it is difficult to derive consistent values for $A_{V}$ if different techniques are applied.

Finally, RNO 1B also shows a discrepancy between our value for $A_{V}$ and that given by Staude \& Neckel (1991). In this case, however, the silicate absorption feature bears evidence for additional superposed emission as is described in the next paragraph. Thus, we attribute at least part of the missing optical depth to an underlying silicate emission feature.

TABLE 5

Mass Fractions of Dust Species with Different Grain Sizes Derived from the Dust Model Fit for FU Ori Shown in Figure 13

\begin{tabular}{|c|c|c|c|c|}
\hline Species & $0.1 \mu \mathrm{m}$ & $1.5 \mu \mathrm{m}$ & $6.0 \mu \mathrm{m}$ & Total \\
\hline Amorphous olivine .................... & 0.16 & 0.20 & 0.21 & 0.57 \\
\hline Amorphous pyroxene................ & 0.00 & 0.00 & 0.43 & 0.43 \\
\hline Crystalline forsterite...................... & $<0.01$ & 0.00 & 0.00 & $<0.01$ \\
\hline Crystalline enstatite .................... & 0.00 & 0.00 & 0.00 & 0.00 \\
\hline 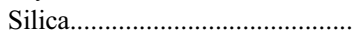 & 0.00 & 0.00 & 0.00 & 0.00 \\
\hline Amorphous carbon....................... & 0.00 & 0.00 & 0.00 & 0.00 \\
\hline 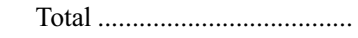 & 0.16 & 0.20 & 0.64 & $\ldots$ \\
\hline
\end{tabular}

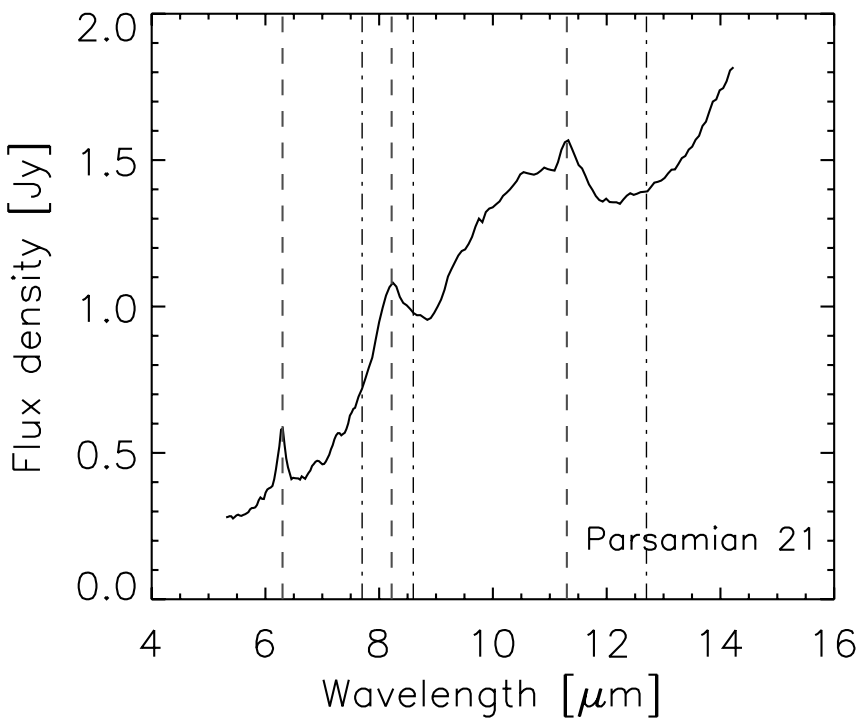

FIG. 14.-Dereddened Spitzer MIR spectrum of Parsamian 21 (assuming $A_{V}=4.0 \mathrm{mag}$ ) illustrating the strong PAH emission features not seen in any other FUor. Note also the steeply rising continuum and the underlying silicate dust emission. The vertical lines indicate the positions of typical PAH emission bands: strong emission is detected at 6.3, 8.2, and $11.3 \mu \mathrm{m}$ (dashed lines), and no emission is seen at $7.7,8.6$, and $12.7 \mu \mathrm{m}$. [See the electronic edition of the Journal for a color version of this figure.]

Not only to measure the depth of the absorption profile, but also to get an idea of the dust composition responsible for the observed absorption, we analyzed the shape of the silicate feature. In Figure 16 we show the optical depths computed or measured for different dust grain compositions. Depending on the composition, the wavelengths of the maximum optical depth change. The most extreme cases are amorphous olivine grains with a size of $0.1 \mu \mathrm{m}$ peaking at a wavelength slightly longward of $9.7 \mu \mathrm{m}$, and amorphous pyroxene grains with the same size peaking around $9.2 \mu \mathrm{m}$ wavelength. As mentioned above, in Table 6 we already give the wavelengths of the maximum optical depths observed toward our sources, and it shows that there is indeed a broad range of values, indicating different dust compositions.

In Figure 17 we plot again the observed optical depth of our objects as shown in Figure 15, but now we overplot one or two of the absorption profiles presented in Figure 16, scaled to the maximum observed optical depth. Whether this scaling is appropriate is difficult to determine, but it shows that most observed features agree reasonably well with one or two of the reference profiles. Three objects (L1551 IRS 5, V346 Nor, and V1735 Cyg) show additional absorption longward of $11 \mu \mathrm{m}$ when compared to the dust features, but at least part of this absorption can be attributed to $\mathrm{H}_{2} \mathrm{O}$ libration bands (e.g., Pontoppidan et al. 2005). V1735 Cyg, RNO $1 \mathrm{C}$, and Z CMa seem to agree best with the dust model computed by Draine (2003) based on the particle size distribution from Weingartner \& Draine (2001). V346 Nor requires shortward of $10 \mu \mathrm{m}$ a mixture of the same model and the dust composition observed toward the Galactic center (Kemper et al. 2004). Longward of $10 \mu \mathrm{m}$ the former model alone fits better. For Reipurth 50 a combination of both models explains the data well over the whole wavelength regime with the Draine (2003) model fitting the short-wavelength part and the Kemper et al. (2004) model fitting the long-wavelength part. L1551 IRS 5 is best fitted with a mixture of small, amorphous pyroxene grains and the model from Draine (2003). The profile of HL Tau agrees extremely well with the Galactic center dust profile from Kemper et al. (2004). To account for a small shift toward shorter wavelengths, a little bit more 

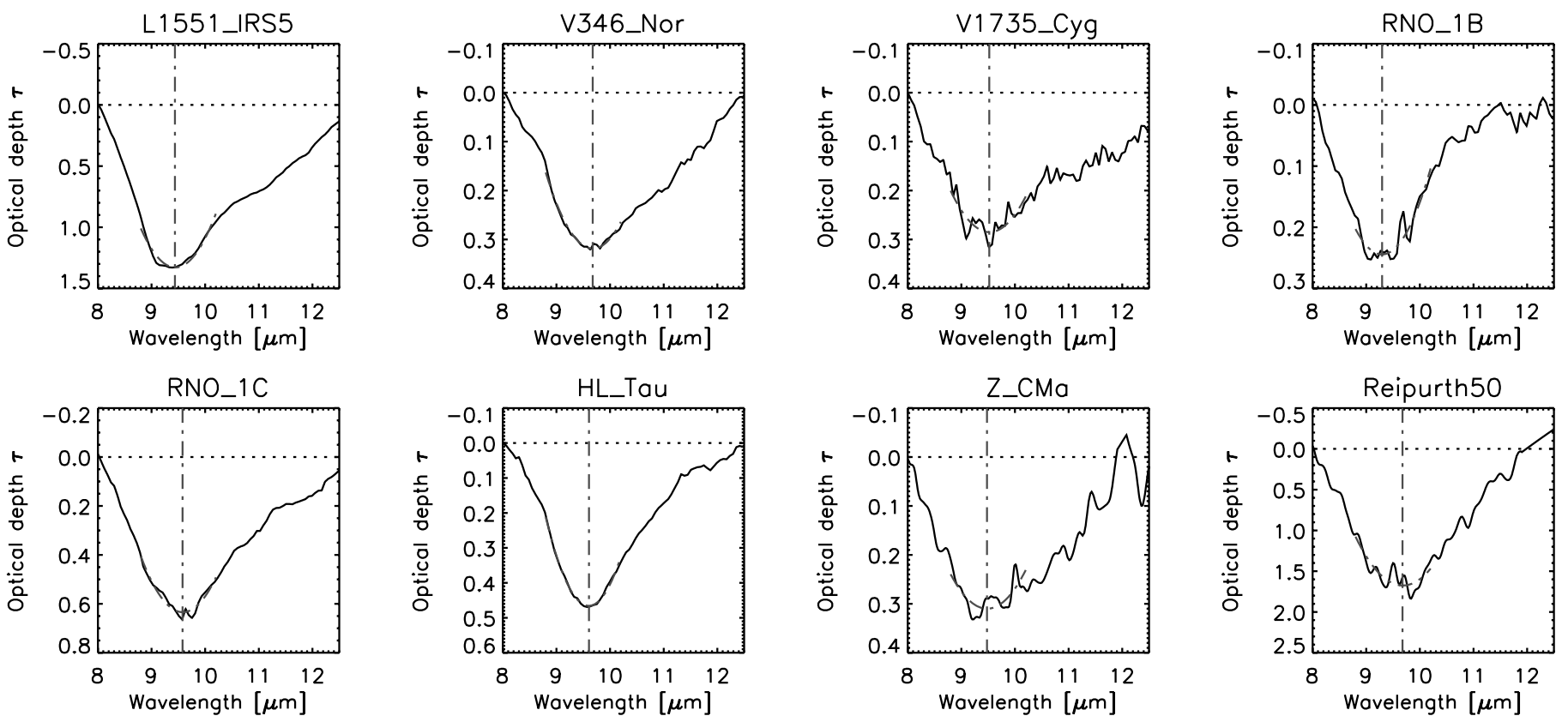

FIG. 15.-Observed optical depths of the $10 \mu \mathrm{m}$ silicate absorption feature (solid line). The dotted line shows the assumed continuum. The vertical dot-dashed lines

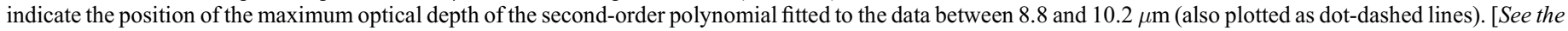
electronic edition of the Journal for a color version of this figure.]

amorphous pyroxene seems to be required, or small uncertainties in the position of the peak absorption from the continuum fit can explain this shift. Finally, the absorption profile of RNO 1B is special in two ways: First, the short-wavelength range is best fitted solely with small, amorphous pyroxene grains. Second, between 10 and $11.5 \mu \mathrm{m}$ the profile shows a significantly lower optical depth than any of the reference profiles. In combination with our findings for the visual extinction (see above), this suggests that RNO 1B shows silicate emission superposed on the absorption feature. Interestingly, the apparent decrease in optical depth longward of $10 \mu \mathrm{m}$ indicates that at these wavelengths the contribution of the emission is relatively larger compared to the other wavelengths in the silicate band. This in turn means that the emission profile does not have the typical shape of the ISM dust feature but a broader and more evolved profile like the other FUor emission profiles shown in Figure 10.

\subsubsection{The $15.2 \mu \mathrm{m} \mathrm{CO}_{2}$ Ice Feature}

The shape of the bending mode profile of $\mathrm{CO}_{2}$ ice around $15.2 \mu \mathrm{m}$ not only allows a detailed analysis of the involved ice inventory but also enables us to derive information about potential ice processing due to heating effects (e.g., Pontoppidan et al. 2005). In Figure 18 we present two reference cases that we use in the following as a benchmark for comparison with the FUor

TABLE 6

Optical Depths and Extinction Values as Derived from the Spectra Shown in Figure 15

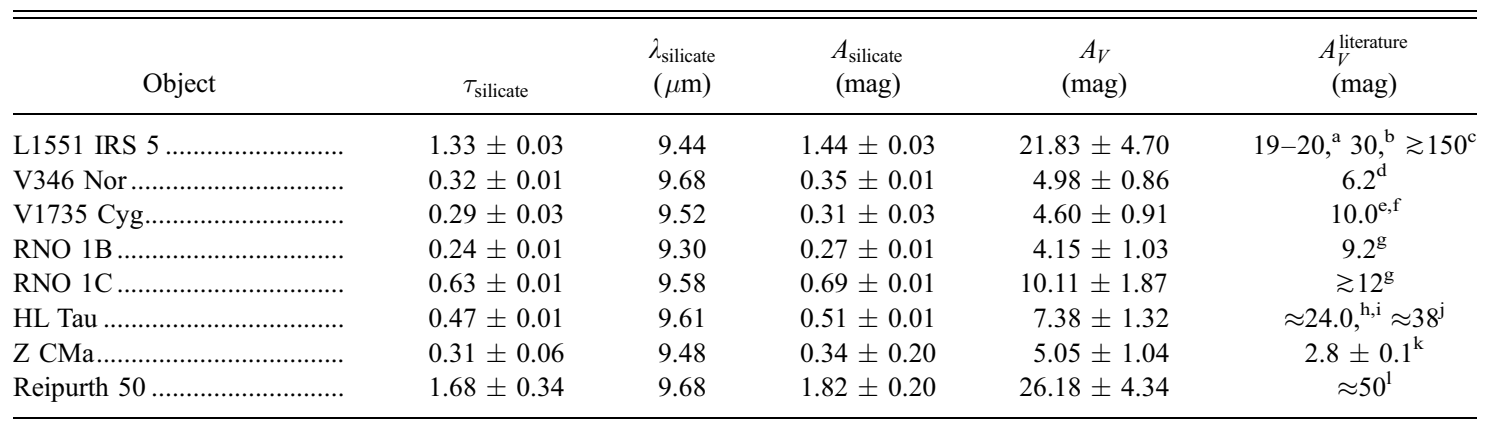

NotE.-The last column lists reference values for $A_{V}$ from the literature.

${ }^{a}$ Snell \& Schloerb (1985).

b Smith et al. (1987).

c Campbell et al. (1988)

d Graham \& Frogel (1985) assuming $E(B-V)=2.0$.

e Sato et al. (1992).

${ }^{f}$ Levreault (1988).

g Staude \& Neckel (1991) based on optical data and assuming spectral type.

h Bergin et al. (2005).

i Close et al. (1997).

j Men'shchikov et al. (1999) derived from radiative transfer model.

k Cohen \& Kuhi (1979) based on optical data and assuming spectral type.

${ }^{1}$ Casali (1991) based on NIR colors, assumed intrinsic color temperatures, and strength of additional ice absorption features. 


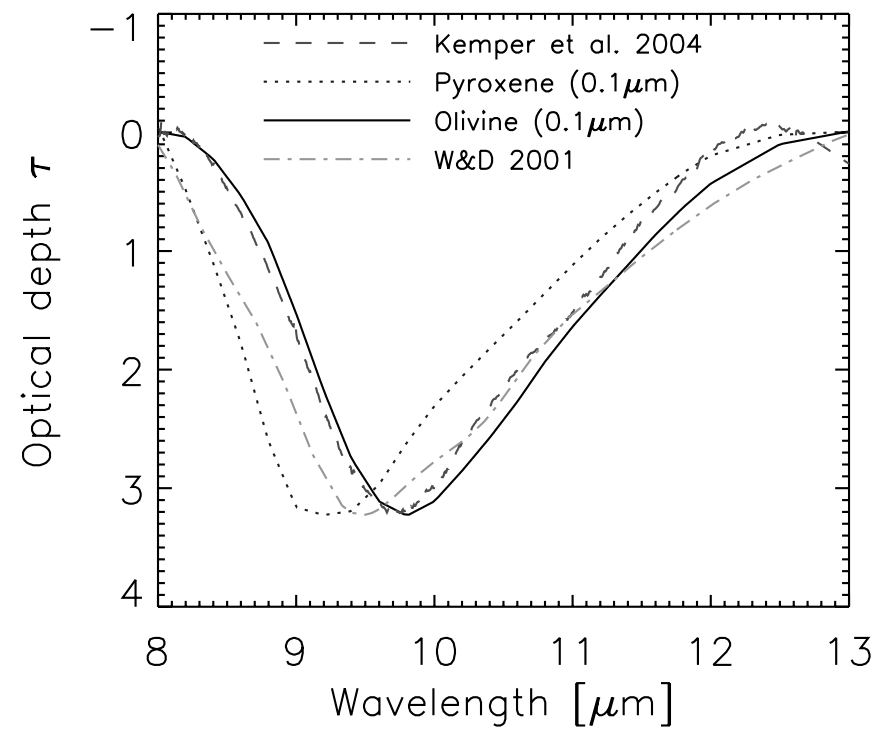

Fig. 16.-Optical depths for different dust grain populations on an arbitrary scale. The solid line shows the profile for $0.1 \mu \mathrm{m}$ sized amorphous olivine grains (Dorschner et al. 1995), the dashed line shows the ISM feature toward the Galactic plane from Kemper et al. (2004), the dot-dashed line is the profile based on the astronomical silicates from Weingartner \& Draine (2001) and Draine (2003), and the dotted line is for $0.1 \mu \mathrm{m}$ sized amorphous pyroxene grains (Dorschner et al. 1995). [See the electronic edition of the Journal for a color version of this figure.]

spectra. The first object in Figure 18 (CK 2) is a highly extincted background star behind the Serpens dark cloud (Knez et al. 2005), and the second object ( $\mathrm{HH} 46$ ) is an embedded low-mass protostar (Boogert et al. 2004).

While the spectrum of CK 2 probes mainly the ice composition of the intervening dark cloud, the spectrum of HH 46 bears information about the immediate environment of the young star. Apart from being slightly broader, the spectrum of $\mathrm{HH} 46$ shows a double-peaked substructure that is caused by crystallization and effective segregation of the $\mathrm{CO}_{2}$ and $\mathrm{H}_{2} \mathrm{O}$ ice involved in the absorption. These processes take place when ice mixtures with concentrations of $\mathrm{CO}_{2} / \mathrm{H}_{2} \mathrm{O} \geq 1$ are heated (Boogert et al. 2004). In space, this phase transition from amorphous to crystalline ice is expected to occur between 50 and $90 \mathrm{~K}$, and thus at higher temperatures than typically found in cold, dark molecular clouds. Thus, the $15.2 \mu \mathrm{m}$ ice feature of $\mathrm{HH} 46$ shows that (at least) part of the ice must already have been heated by the embedded protostar. In fact, Boogert et al. (2004) fitted the feature with a two-component ice model based on laboratory spectra, with one component being highly processed polar ice with a laboratory temperature of $T_{\text {lab }}=125 \mathrm{~K}$ and the other component being an $\mathrm{H}_{2} \mathrm{O}$-rich, $\mathrm{CH}_{3} \mathrm{OH}$-deficient cold ice with $T_{\text {lab }}=10 \mathrm{~K}^{10}$ The spectrum of CK 2 was fitted by Knez et al. (2005) solely with cold ice components. They used a polar mixture of $\mathrm{H}_{2} \mathrm{O}: \mathrm{CO}_{2}=$ $1: 1$ and $\mathrm{H}_{2} \mathrm{O}: \mathrm{CO}_{2}=10: 1$ at $10 \mathrm{~K}$ with a ratio of $2: 1$ and an additional apolar component of $\mathrm{CO}: \mathrm{N}_{2}: \mathrm{CO}_{2}=100: 50: 20$ at $30 \mathrm{~K}$. The overall polar fraction was assumed to be $78 \%$.

In Figure 19 we present the $15.2 \mu \mathrm{m}$ features observed toward our FUor sample. To derive the optical depths, we fitted the continuum with a straight line fixed around 14.65 and $16.3 \mu \mathrm{m}$. For each object we overplot the (scaled) spectrum of either HH 46 or CK 2 depending on the shape of the profile. While the profiles of V1647 Ori and V346 Nor agree better with the profile of CK 2 (the spectrum representing unprocessed ice), L1551 IRS 5, RNO 1B and 1C, and HL Tau show evidence for a double-peaked substructure and thus heating effects and processed ice. The comparatively bad quality of the V1735 Cyg spectrum does not allow a solid comparison to either reference spectrum. It is noteworthy that the spectrum of V1647 Ori is almost an exact copy of CK 2, indicating that the ice composition is mostly identical. Based on the fitted reference spectrum, we computed also the optical depth $\tau_{15.2 \mu \mathrm{m}}$ for each object and summarized the results in Table 7. The errors are based on the $1 \sigma$ uncertainties in the observed spectra.

10 The presence of $\mathrm{CH}_{3} \mathrm{OH}$ in the ice feature can potentially be traced by the shape of the long-wavelength wing of the $\mathrm{CO}_{2}$ profile, showing additional absorption if $\mathrm{CH}_{3} \mathrm{OH}$ is present in higher abundances.
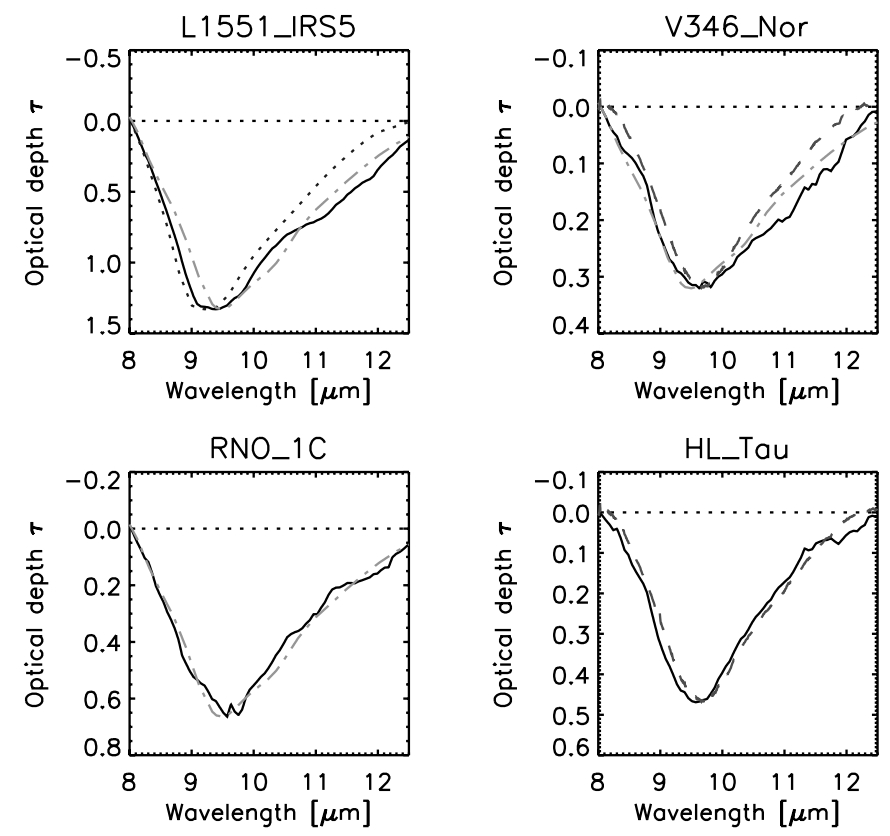
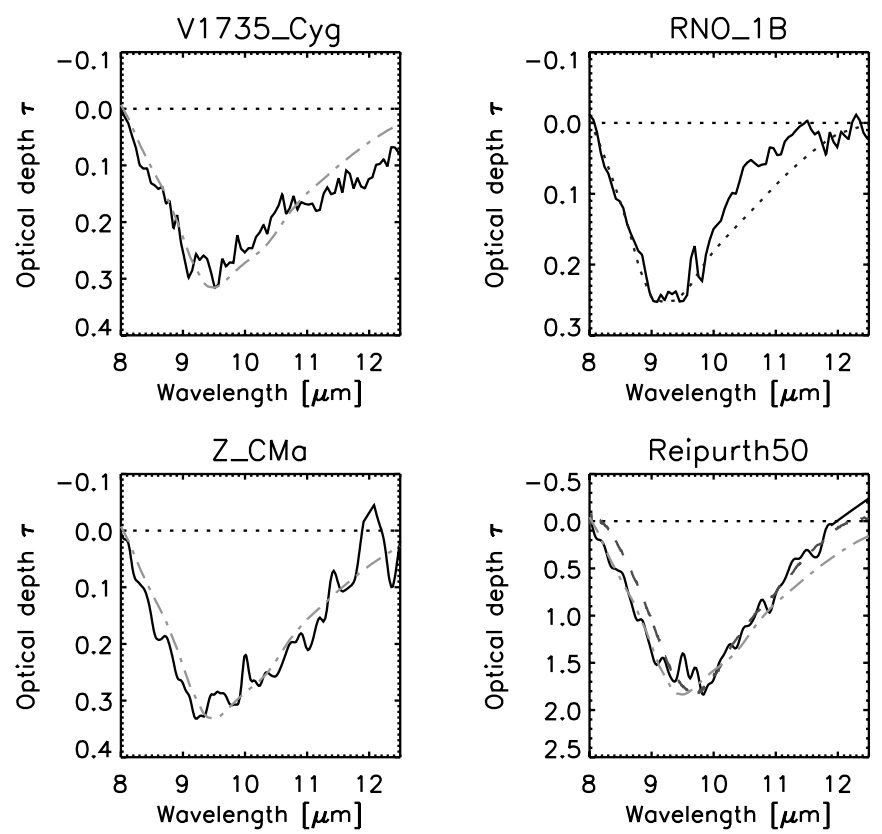

FIG. 17.- Same observed spectra as in Fig. 15 (solid lines), but now overplotted with reference spectra with different dust compositions (same line style code as in Fig. 16). [See the electronic edition of the Journal for a color version of this figure.] 


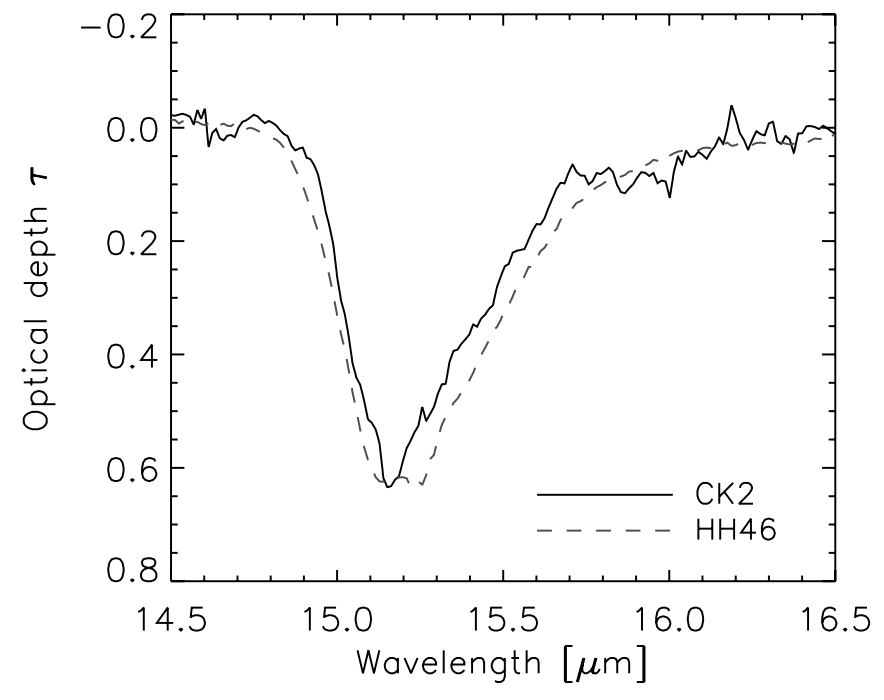

FIG. 18.-Comparison of the $15.2 \mu \mathrm{m} \mathrm{CO}_{2}$-ice feature between the heavily extincted background object CK 2 (Knez et al. 2005) and the embedded young source HH 46 (Boogert et al. 2004). Due to higher temperatures in the vicinity of the embedded protostar, crystallization occurs and a double-peaked substructure appears characteristic of the pure $\mathrm{CO}_{2}$ matrix (Ehrenfreund et al. 1998). [See the electronic edition of the Journal for a color version of this figure.]

\subsubsection{The 6.0 and $6.8 \mu \mathrm{m}$ Ice Features}

Although frequently observed toward high- and low-mass sources, the two well-known ice features at 6.0 and $6.8 \mu \mathrm{m}$ are quite complex and difficult to interpret. Certainly, a large fraction of the optical depth of the $6.0 \mu \mathrm{m}$ band can be attributed to $\mathrm{H}_{2} \mathrm{O}$ ice, but also other species might contribute to this absorption feature (Keane et al. 2001). For instance, slightly shortward, at roughly $5.85 \mu \mathrm{m}$, an additional absorption shoulder is sometimes superposed (e.g., Pontoppidan et al. 2005; Keane et al. 2001) for which formaldehyde $\left(\mathrm{H}_{2} \mathrm{CO}\right)$ and formic acid $(\mathrm{HCOOH})$ are theoretical candidates. Without any additional information (e.g., the $3.08 \mu \mathrm{m}$ band of $\mathrm{H}_{2} \mathrm{O}$ or the $3.47 \mu \mathrm{m}$ band of $\mathrm{H}_{2} \mathrm{CO}$ ) it is thus difficult to determine the true water ice content in the $6.0 \mu \mathrm{m}$ band.

An additional absorption feature at $6.85 \mu \mathrm{m}$ is often observed toward protostars (e.g., Keane et al. 2001), but also toward the extincted background star CK 2 (Knez et al. 2005) and the edgeon disk CRBR 2422.8-3423 (Pontoppidan et al. 2005). Although a final identification of this band has yet to be provided, $\mathrm{NH}_{4}^{+}$ seems to be one of the most promising candidates (Schutte \& Khanna 2003; Pontoppidan et al. 2005). However, van Dishoeck (2004), for example, mentions also methanol $\left(\mathrm{CH}_{3} \mathrm{OH}\right)$ as a potential carrier of this absorption band.

Given all these uncertainties, we restrict ourselves in this section to the computation of the optical depth of both of the abovementioned absorption bands. Like in the previous section, we assumed a straight line for the continuum anchored at 5.4 and $7.6 \mu \mathrm{m}$. To derive the optical depths, we then fitted a polynomial of fourth order to both absorption dips to eliminate the noise in the spectra. Figure 20 shows the observed spectra between 5.5 and $7.5 \mu \mathrm{m}$ on an optical depth scale (solid lines) with the resulting fits overplotted (dot-dashed lines). The computed optical depth for each band and each object is given in Table 7. Like in the previous section, the errors are based on the $1 \sigma$ uncertainties in the observed spectra.

\subsection{The Fading of OO Ser and V1647 Ori}

For two objects (OO Ser and V1647 Ori) we have multiepoch data and can derive some conclusions on the variability of these objects. As illustrated in Figure 4, we fitted a straight line to the data of OO Ser between 15 and $30 \mu \mathrm{m}$ to estimate the decay in flux density observed over the five epochs (see Table 1). This wavelength range was chosen since in this regime all spectra are still relatively clear of artifacts and spikes partly seen at longer wavelengths. In Table 8 we summarize the flux density level at 20 and $30 \mu \mathrm{m}$ at each epoch and give also the slope measured between 15 and $30 \mu \mathrm{m}$. The errors are derived from the goodness
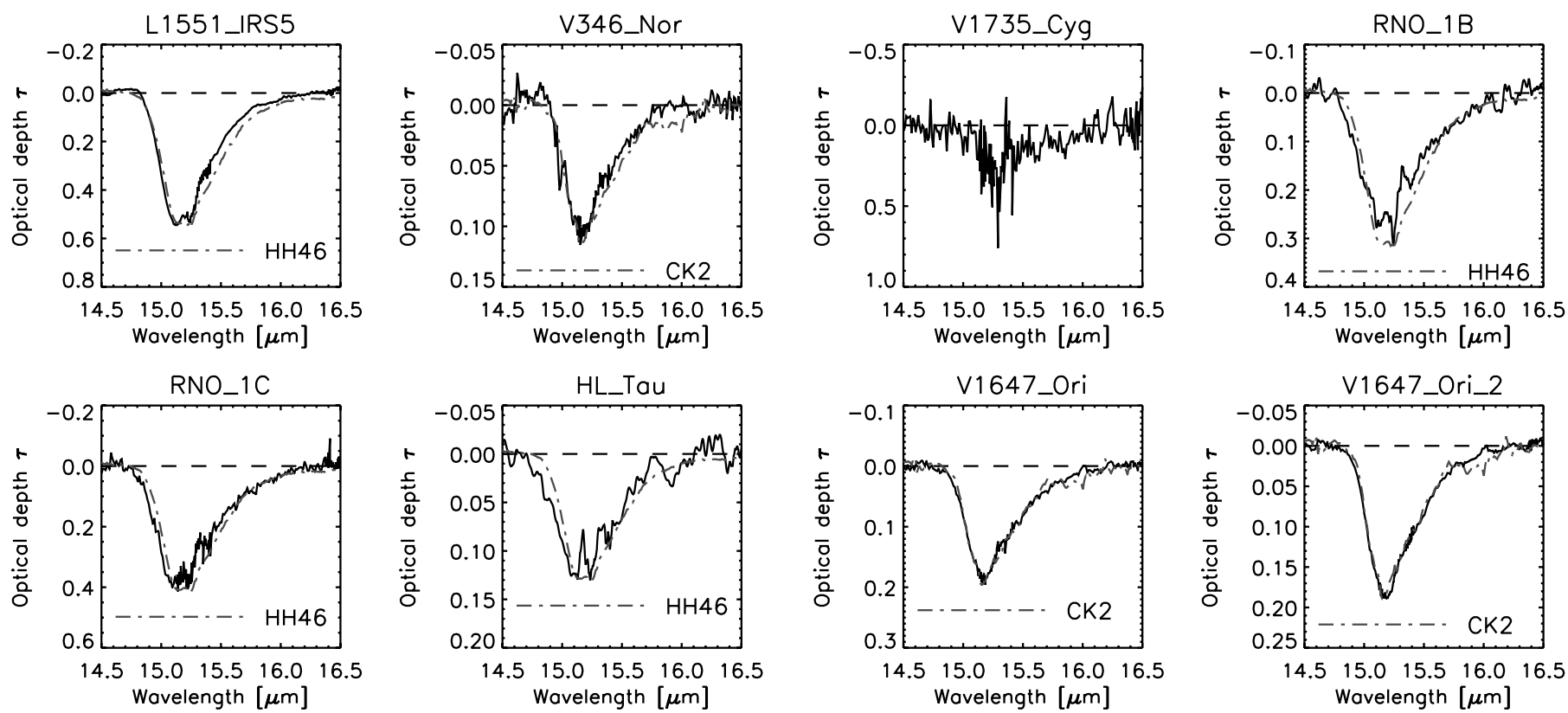

FIG. 19.- The $15.2 \mu \mathrm{m} \mathrm{CO}_{2}$-ice feature observed toward some of our objects (solid lines). The spectra of HL Tau, RNO 1B, and V1735 Cyg were smoothed by a

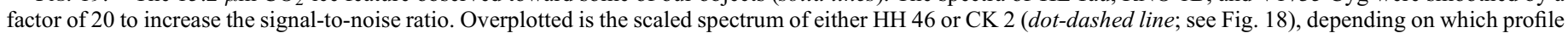

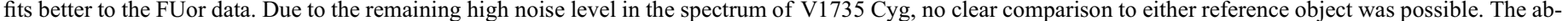

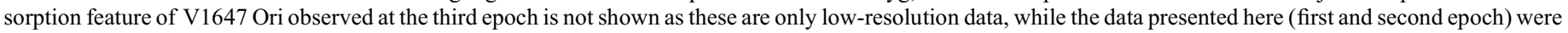
taken with the high-resolution spectrograph. [See the electronic edition of the Journal for a color version of this figure.] 
TABLE 7

Optical Depths of the Main Ice Features at 6.0, 6.85, and $15.2 \mu \mathrm{m}$

\begin{tabular}{|c|c|c|c|}
\hline Object & $\tau_{6.0 \mu \mathrm{m}}$ & $\tau_{6.85 \mu \mathrm{m}}$ & $\tau_{15.2 \mu \mathrm{m}}$ \\
\hline L1551 IRS $5 \ldots \ldots \ldots \ldots \ldots \ldots$ & $0.51 \pm 0.01$ & $0.47 \pm 0.01$ & $0.54 \pm 0.05$ \\
\hline 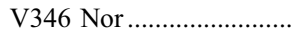 & $0.05 \pm 0.01$ & $0.06 \pm 0.01$ & $0.12 \pm 0.02$ \\
\hline V1735 Cyg.... & $0.05 \pm 0.02$ & $0.09 \pm 0.01$ & $\approx 0.3$ \\
\hline RNO $1 \mathrm{~B} \ldots \ldots$. & $0.20 \pm 0.01$ & $0.18 \pm 0.01$ & $0.32 \pm 0.04$ \\
\hline RNO $1 \mathrm{C} \ldots \ldots \ldots . . .$. & $0.35 \pm 0.01$ & $0.26 \pm 0.01$ & $0.41 \pm 0.02$ \\
\hline 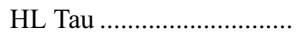 & $0.10 \pm 0.01$ & $0.07 \pm 0.01$ & $0.13 \pm 0.06$ \\
\hline V1647 Ori .......................... & $0.07 \pm 0.01$ & $0.06 \pm 0.01$ & $0.20 \pm 0.02$ \\
\hline 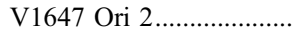 & $0.10 \pm 0.01$ & $0.08 \pm 0.01$ & $0.19 \pm 0.02$ \\
\hline 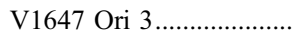 & $0.09 \pm 0.01$ & $0.07 \pm 0.01$ & a \\
\hline
\end{tabular}

a Only low-resolution data. of the fit to the data. Between the first observations on 1996 April 14 and the last observations carried out on 1997 September 22, the flux densities decreased to roughly $50 \%$ of the initial values. These data demonstrate that OO Ser faded rapidly over relatively short timescales and that it might be an intermediate object between a typical FUor and an EXor having fading timescales of several decades and months, respectively. Based on photometric monitoring at infrared wavelengths, Kóspál et al. (2007) came to a similar conclusion and predicted that OO Ser should return to its preoutburst luminosity not before 2011 .

In Table 8 we also summarize the flux densities of V1647 Ori observed at 8,20 , and $30 \mu \mathrm{m}$ at three different epochs. The errors are taken directly from the Spitzer spectra. It shows that also this object faded significantly over a period of less than 5 months between 2004 October and $2005 \mathrm{March}$. In addition, short time variations in the flux levels seem to be present, as between the two
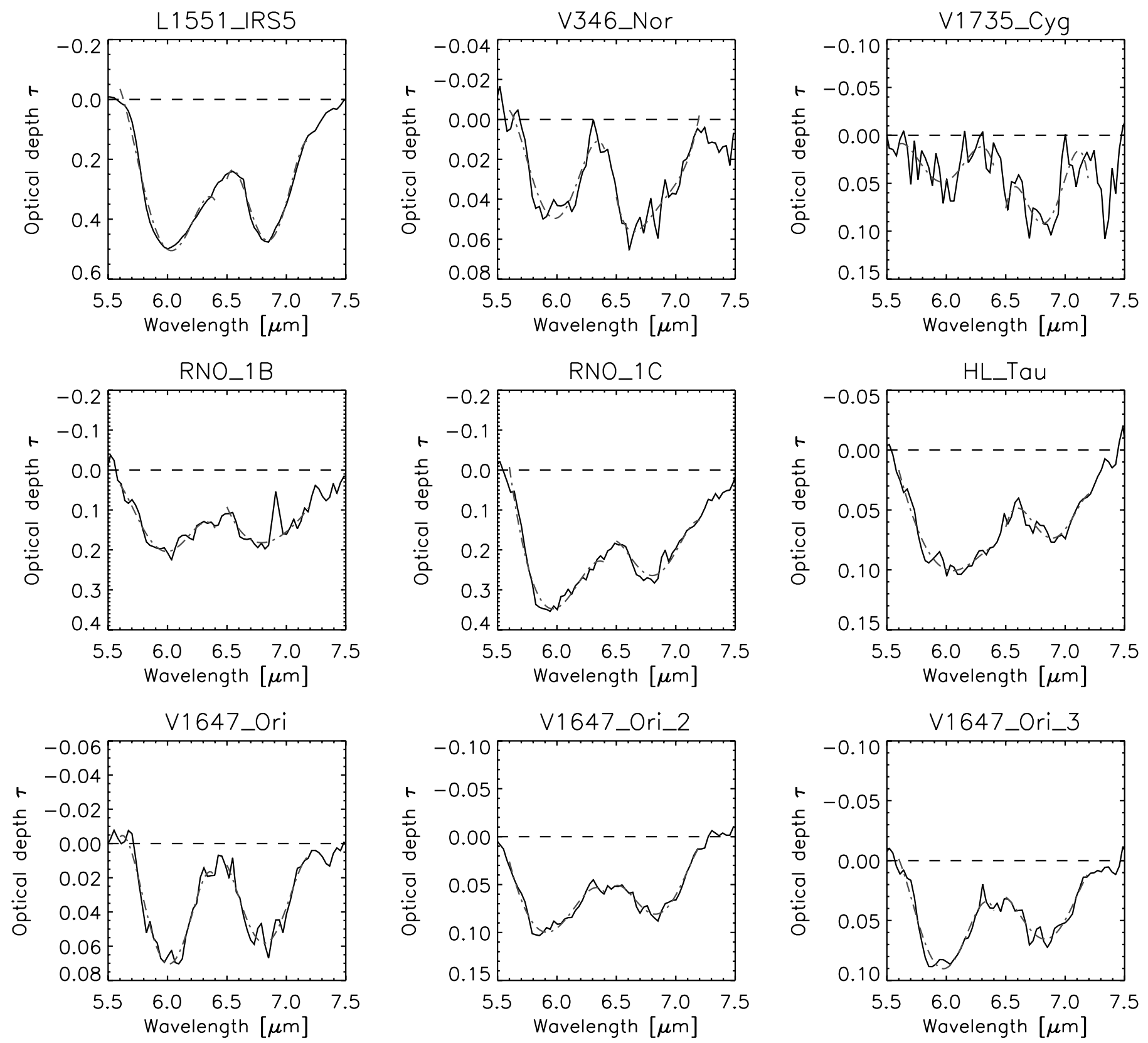

FIG. 20.- The 6.0 and $6.85 \mu \mathrm{m}$ ice bands observed toward some of our objects (solid lines). Overplotted are the polynomial fits of fourth order (dot-dashed line) to determine the optical depths of the absorption bands. [See the electronic edition of the Journal for a color version of this figure.] 
TABLE 8

Flux Densities and the Slope of the SED

\begin{tabular}{|c|c|c|c|c|}
\hline Date & $\begin{array}{c}\text { Flux Density at } 8 \mu \mathrm{m} \\
\text { (Jy) }\end{array}$ & $\begin{array}{c}\text { Flux Density at } 20 \mu \mathrm{m} \\
\text { (Jy) }\end{array}$ & $\begin{array}{c}\text { Flux Density at } 30 \mu \mathrm{m} \\
\text { (Jy) }\end{array}$ & $\begin{array}{c}\text { Slope } \\
\left(\text { Jy } \lambda^{-1}\right)\end{array}$ \\
\hline \multicolumn{5}{|c|}{ OO Ser } \\
\hline 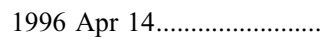 & $\ldots$ & $16.5 \pm 1.8$ & $43.0 \pm 2.3$ & $2.65 \pm 0.04$ \\
\hline 1996 Oct 24 & $\ldots$ & $15.2 \pm 2.1$ & $41.2 \pm 2.6$ & $2.61 \pm 0.05$ \\
\hline 1997 Mar 08 .......................... & $\ldots$ & $12.8 \pm 1.2$ & $32.5 \pm 1.5$ & $1.98 \pm 0.03$ \\
\hline 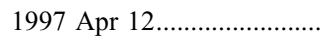 & $\ldots$ & $11.9 \pm 1.5$ & $31.1 \pm 1.8$ & $1.92 \pm 0.04$ \\
\hline 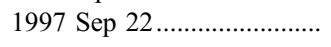 & $\ldots$ & $8.8 \pm 1.2$ & $23.9 \pm 1.4$ & $1.52 \pm 0.03$ \\
\hline \multicolumn{5}{|c|}{ V1647 Ori } \\
\hline 2004 Oct $20 \ldots \ldots \ldots$ & $3.95 \pm 0.02$ & $11.56 \pm 0.16$ & $16.52 \pm 0.51$ & $\ldots$ \\
\hline 2005 Mar 11 ......................... & $2.49 \pm 0.01$ & $7.21 \pm 0.20$ & $10.35 \pm 0.24$ & $\ldots$ \\
\hline 2005 Mar $24 \ldots \ldots \ldots \ldots$ & $3.04 \pm 0.01$ & $7.82 \pm 0.17^{\mathrm{a}}$ & $10.62 \pm 0.05^{\mathrm{a}}$ & $\ldots$ \\
\hline
\end{tabular}

Notes.-Upper rows: Flux densities at 20 and $30 \mu \mathrm{m}$ and the slope of the SED between 15 and $30 \mu \mathrm{m}$ for OO Ser observed at five different epochs. Lower rows: Flux densities at 8, 20, and $30 \mu \mathrm{m}$ for V1647 Ori at three different epochs.

${ }^{\mathrm{a}}$ Low-resolution data.

epochs in 2005 March the object became slightly brighter again. In consequence, these data support the assumption that the outburst of V1647 Ori may also be intermediate between FUor- and EXor-type events similar to OO Ser (Muzerolle et al. 2005; AcostaPulido et al. 2007).

\subsection{Additional Emission Lines}

For completeness we show in Figure 21 absorption and emission lines identified in the high-resolution regime of the Spitzer spectra, part of which are difficult to identify in Figures 7 and 8. Already Green et al. (2006) noted the [S III] emission lines at 18.7 and $33.4 \mu \mathrm{m}$ in the spectrum of V1515 Cyg and argued that they originate from extended emission in the region and not from the object itself. The spectra of RNO 1B and RNO 1C show evidence for $\mathrm{H}_{2}$ quadrupole emission around $17 \mu \mathrm{m}$ and additional $\mathrm{H}_{2}$ lines in the low-resolution part of the spectrum at shorter wavelengths. In Quanz et al. (2007) we analyzed these emission lines in detail and concluded that they are related to shocks within a molecular
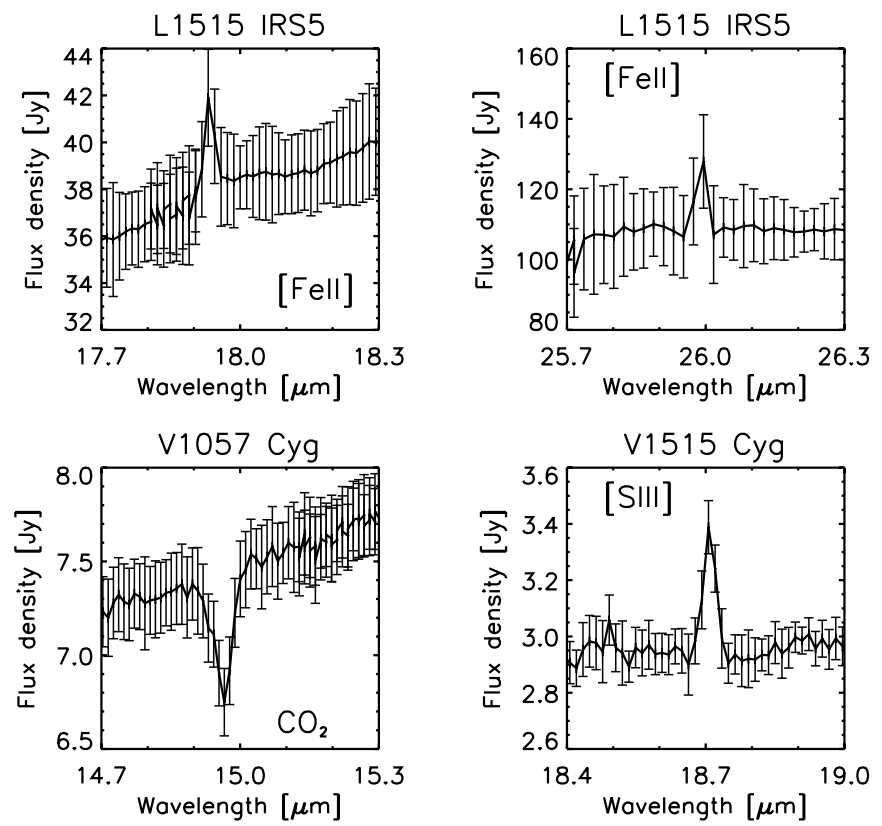

outflow powered by the nearby embedded object IRAS $00338+$ 6312. [Fe II] lines around $17.9 \mu \mathrm{m}$ are present in the spectra of L1551 IRS 5 and RNO 1B, and L1551 IRS 5 shows also the [Fe II] line near $26.0 \mu \mathrm{m}$. The [Fe II] lines of L1551 IRS 5 were already detected in the ISO SWS spectrum of this source and attributed to hot and dense material located close to the root of the outflow (White et al. 2000). The line intensities did not fit the predictions from shock models. Concerning the [Fe II] line in the spectrum of RNO 1B, it seems likely that it arises in the outflow shocks that also excite the $\mathrm{H}_{2}$ emission lines discussed in Quanz et al. (2007). Finally, V1057 Cyg shows gaseous $\mathrm{CO}_{2}$ absorption slightly shortward of $15.0 \mu \mathrm{m}$.

\section{DISCUSSION}

\subsection{Two Categories of FUors}

The results presented here suggest that the sample of FUors can be divided into two categories based on the observational
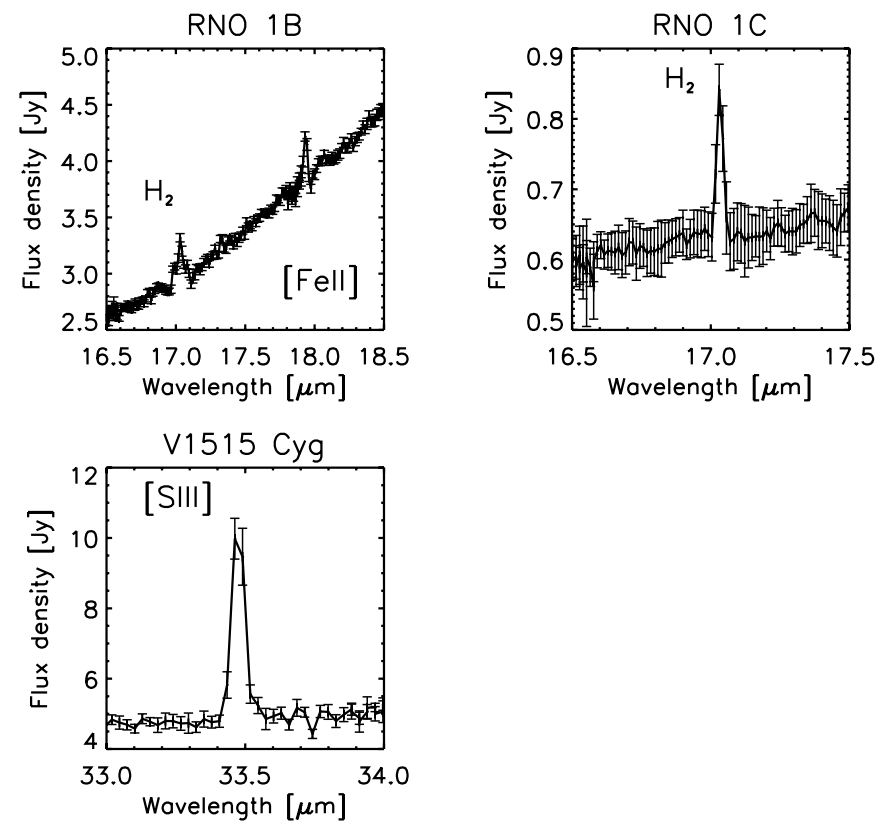

FIG. 21.-Zoom into emission lines and absorption lines detected in the high-resolution part of the spectra of some objects (see text). 

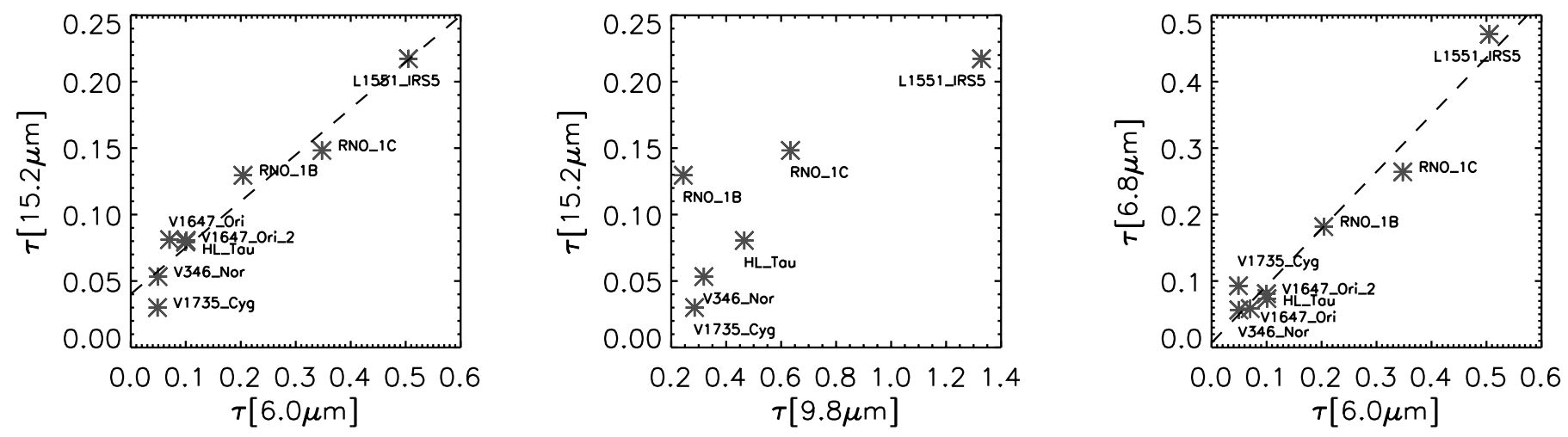

FIG. 22.-Correlation between the optical depths of ice features at 15.2 and $6.0 \mu \mathrm{m}($ left $)$, ices and silicates at 15.2 and $9.8 \mu \mathrm{m}($ middle $)$, and ice features at 6.8 and $6.0 \mu \mathrm{m}$

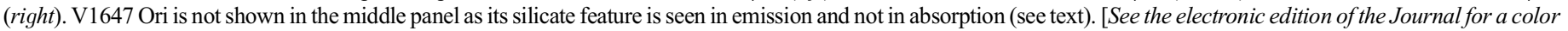
version of this figure.]

appearance of the $10 \mu \mathrm{m}$ silicate feature. We decided to call the absorption feature objects "Category 1" FUors (nine objects in Table 3 ) and the emission feature objects "Category 2" FUors (six objects in Table 3). In the following we discuss the properties of the two categories in more detail.

\subsubsection{Category 1 FUors: Silicate and Ice Absorption Features}

In general it is possible to observe the silicate feature in absorption if the circumstellar disk surrounding a young object is seen close to edge-on. Men'shchikov \& Henning (1997) showed that typically for a disk with an opening angle of $20^{\circ}$ between the upper and lower disk surface the silicate feature appears in absorption only if the disk inclination is $\$ 10^{\circ}$ from edge-on. Given the number of objects showing silicate absorption compared to the number of objects with silicate emission and assuming a random distribution of the orientation of the accretion disks, it is unlikely that all Category 1 FUors are seen edge-on. Rather, these objects are still more deeply embedded in their molecular envelopes, covering a larger solid angle than the edge-on disk alone. These envelopes cause the ice and dust absorption features.

Figure 17 illustrates that all but one silicate absorption band are fitted best either with the silicate composition observed toward the Galactic center by Kemper et al. (2004) or with the astronomical silicates from Weingartner \& Draine (2001) and Draine (2003). ${ }^{11}$ This finding shows that all absorption features can be fitted with small, amorphous silicates and hence the extinction is caused by pristine and not processed dust. Only the spectrum of RNO 1B is difficult to fit with any ISM dust composition, but, as already mentioned, there are strong hints that the absorption feature is altered by a superposed emission feature.

In Figure 22 we show the observed optical depths of the ices and the silicate feature in a scatter plot to search for any correlation. Although the range of optical depths we probe here is limited, it seems that at least for the ices there seem to be correlations (Fig. 22, left and right panels). Fitting a straight line to the data, we find

$$
\tau_{15.2 \mu \mathrm{m}}=(0.349 \pm 0.037) \tau_{6.0 \mu \mathrm{m}}+(0.040 \pm 0.009)
$$

and

$$
\tau_{6.8 \mu \mathrm{m}}=(0.858 \pm 0.067) \tau_{6.0 \mu \mathrm{m}}+(0.007 \pm 0.016) .
$$

\footnotetext{
${ }^{11}$ L1551 IRS 5 requires a fraction of additional amorphous pyroxene grains.
}

This implies that the physical and chemical conditions within the envelopes and clouds causing the absorption are similar. A more detailed analysis, e.g., the determination of different ice abundances relative to water ice, is beyond the scope of this work. For such an analysis the water ice feature around $3 \mu \mathrm{m}$ is required as it suffers less from additional contributions of other ice species compared to the $6 \mu \mathrm{m}$ feature.

For a correlation between the optical depths of the silicates and ices the situation is slightly different because, as we have already pointed out, the silicate feature can be influenced by superposed emission. This is shown nicely in the middle panel of Figure 22, where RNO 1B is shifted with respect to the other objects.

Finally, it is interesting to note that the objects showing evidence for ice processing in Figure 19 tend to show higher optical depths in the ice features than the other sources. This might suggest that the extinction for the latter objects (V346 Nor, V1735 Cyg) might be caused by ices somewhere in the line of sight to the source, rather than by material related to the young star. This might also explain why V1647 Ori shows weak silicate emission, but ice absorption: the extinction is caused by cold foreground material, reflected also in the observed high value for $A_{V}$ (see caption of Fig. 10). Ground-based observations find the spectrum to be flat in the $10 \mu \mathrm{m}$ regime (Ábrahám et al. 2006). However, the sensitivity of Spitzer allows the detection of a weak feature resulting from an extinguished but intrinsically strong silicate emission band.

\subsubsection{Category 2 FUors: The Silicate Emission Feature and Its Dust Composition}

As presented in Figure 10 and described in the related section, the emission profiles bear evidence for dust grain processing. Even after the correction for apparent interstellar extinction, the shape of the silicate profile differs from that of typical ISM dust. Like for T Tauri stars and the slightly more massive HAeBes, the origin of the emission feature in the spectra of the FUors is the heated surface layer of the accretion disk. The apparent grain processing is believed to be only possible in circumstellar disks and not in the less dense circumstellar envelopes. While the emission layer for T Tauri stars and HAeBes is mainly heated be the central stellar object, for FUors the hot inner parts of the accretion disk itself can act as the main illuminating source (e.g., Lachaume 2004). Due to the high accretion rates of FUors, those inner regions are extremely hot and account for a significant fraction of the total flux even at optical wavelengths (Quanz et al. 2006). Furthermore, accretion disk models with a flared geometry were not only able to explain the SEDs of FU Ori and Bran 76 (Green 
et al. 2006), but they could also reproduce interferometric observations in the NIR (Malbet et al. 2005) and MIR (Quanz et al. 2006). V1057 Cyg and V1515 Cyg show more emission at longer infrared wavelengths than the previous objects, which can be accounted for assuming a remnant infalling envelope in addition to the accretion disks. This is supported by the results from $K$-band interferometry (Millan-Gabet et al. 2006), where envelopes are required to explain the low NIR visibilities.

Concerning the dust composition, it is interesting to note that, given the low peak-overcontinuum ratio illustrated in Figure 12, grain growth must have already set in. This is supported by the results of our dust model fit. Furthermore, the spectra do not show evidence for crystalline dust particles. The mass fraction of crystalline particles in the dust model computed for the spectrum of FU Ori was negligible (see Table 5). Since strength and shape of the silicate feature of FU Ori are comparable to those of Bran 76 and V1515 Cyg (Fig. 11), the dust composition in all objects is similar. The spectrum of V1057 Ori is even broader and less pronounced than the other spectra, indicating even larger grains in the disk surface layer. The intrinsic feature of V1647 Ori is stronger than those of the other FUors, but no prominent signs of crystalline silicates are present either. ${ }^{12}$

As already mentioned in Quanz et al. (2006), there are several reasons for which stronger crystalline features could have been expected to be detected. The high disk accretion rates should ease the detection of crystalline particles in two ways: (1) high accretion rates lead to high disk temperatures (Bell et al. 1997), which in turn should increase the amount of crystalline particles produced by annealing processes at $T \geq 800 \mathrm{~K}$; and (2) an increase in the accretion rate should also increase the radial and vertical mixing in the disk (Gail 2001), transporting the crystalline particles farther out and to the disk surface, where they can be detected by means of MIR spectroscopy. However, neither in the innermost disk regions probed with MIR interferometry (Quanz et al. 2006) nor in the Spitzer spectra presented here in Figure 10 or at longer wavelengths in Figure 7 do we see any striking evidence for crystallinity. This means that either those grains do not exist in large amounts in these disks or they are somehow hidden.

The disks of FUors are different from those of $\mathrm{T}$ Tauri or Herbig star disks in the sense that in the radially innermost regions of FUor disks the luminosity is accretion dominated ( $\mathrm{T}$ Tauri and Herbig star disks are irradiation dominated everywhere). At larger radii, also FUor disks are irradiation dominated, albeit the main heating source may not be the central star itself, but rather the hot inner disk regions close to the star where accretion dominates (e.g., Lachaume 2004). Disk regions that are irradiation dominated have a surface layer that is warmer than the underlying disk interior; such an "inverted" temperature profile causes dust features to appear in emission. In the accretion-dominated regions, the main heating source is the release of gravitational energy in the disk midplane. Here the disk interior is at least as hot as (and likely hotter than) the disk surface, causing dust features to be effectively hidden, or even to appear in absorption. ${ }^{13}$ Thus, it is conceivable that there are significant amounts of crystalline silicates present in the hot inner disk regions of our targets, even if they do not show up prominently in the spectra.

But what about crystalline silicates further from the central object, in irradiation-dominated regions? Given the high accre-

\footnotetext{
12 The spectrum of Parsamian 21 does show a prominent feature at $11.3 \mu \mathrm{m}$ but we attribute most of the related flux to PAH emission and not to crystalline silicates (see $\S 3.2 .3$ ).

${ }^{13}$ The gas absorption features between 5 and $8 \mu \mathrm{m}$ described in $\S 3.2 .4$ likely originate in this hot disk region.
}

tion luminosity in our objects, it seems unavoidable that large amounts of crystalline silicates are produced in the innermost disk regions. In addition to this, crystalline silicates may be produced in situ at large radii in shocks (Harker \& Desch 2002) or electric discharges (Pilipp et al. 1998; Desch \& Cuzzi 2000). If the disk is well mixed in the vertical direction, the crystalline silicates should be present in the surface layer of the disk at large ( $~$ few AU) radii and show up in emission, which they evidently do not (at least, to a much lesser extent than in many T Tauri and Herbig Ae/Be star disks, which are more evolved). This inevitably leads to the conclusion that crystalline silicates are not abundant in the surface layer of FUor disks at radii of more than a few AU from the central object, contrary to expectations.

At this point, we have no proper explanation for this observation. In order to animate the discussion, we post the following idea but stress that at this point this is mere speculation. The crystalline silicates are not present in the surface layer at larger radii but may be present in the disk interior, where they are expected to be formed by virtue of the processes mentioned before. Thus, we need to explain why the crystalline silicates do not get mixed in the vertical direction all the way to the disk surface. One possibility is that the crystalline particles are somehow less well coupled to the gas, possibly because they are more compact (or less "fluffy") than the unprocessed dust particles. This may cause the selective settling of crystalline dust.

A second possibility is the following. The accretion rate in FUors is high, suggesting that the disk is still being supplied with fresh material from the maternal envelope. This material, which is thought to contain exclusively small, amorphous dust particles, will in part "rain" onto the disk surface at larger radii. If the rate at which this material falls onto the disk is higher than the rate at which it is mixed through the disk in the vertical direction, the disk may be covered by a "blanket" of pristine material, effectively hiding the more processed material present in the disk interior. It is unclear whether this idea can be harmonized with the observation that the dust we see at the disk surface has undergone significant grain growth. This issue clearly calls for a much more detailed and quantitative investigation, which is beyond the scope of the present work.

\subsection{Unifying the Two Categories of FUors: An Evolutionary Sequence}

The spectral properties of the sample presented here draw a rather inhomogeneous picture of the group of FU Orionis objects. However, as most of them are convincingly classified as FUors, they do share some common observational and physical properties. In the following, we present an idea how the apparent differences can be explained within a unified paradigm of FUor objects.

Already 20 years ago Herbig (1977) and Hartmann \& Kenyon (1985) suggested that each FUor might undergo several outbursts. More recent theoretical models show that gravitational instabilities in the accretion disk, driven by continuing infall from a remnant envelope, can account for intense bursts of high accretion rates that are intersecting more prolonged, quiescent periods of low accretion (Vorobyov \& Basu 2006; Boley et al. 2006). After several of these outbursts have occurred within a time span of several times $10^{5} \mathrm{yr}$, the envelope, which is the trigger of the disk instabilities, vanishes, and the object enters finally a state of permanently low accretion. If one relates this final phase to the classical T Tauri phase of low-mass YSOs, then FUors are younger than classical T Tauri stars as already pointed out by Weintraub et al. (1991) and Sandell \& Weintraub (2001).

Kessler-Silacci et al. (2005) analyzed the $10 \mu \mathrm{m}$ silicate feature of 34 young stars and confirmed that the evolution of the 


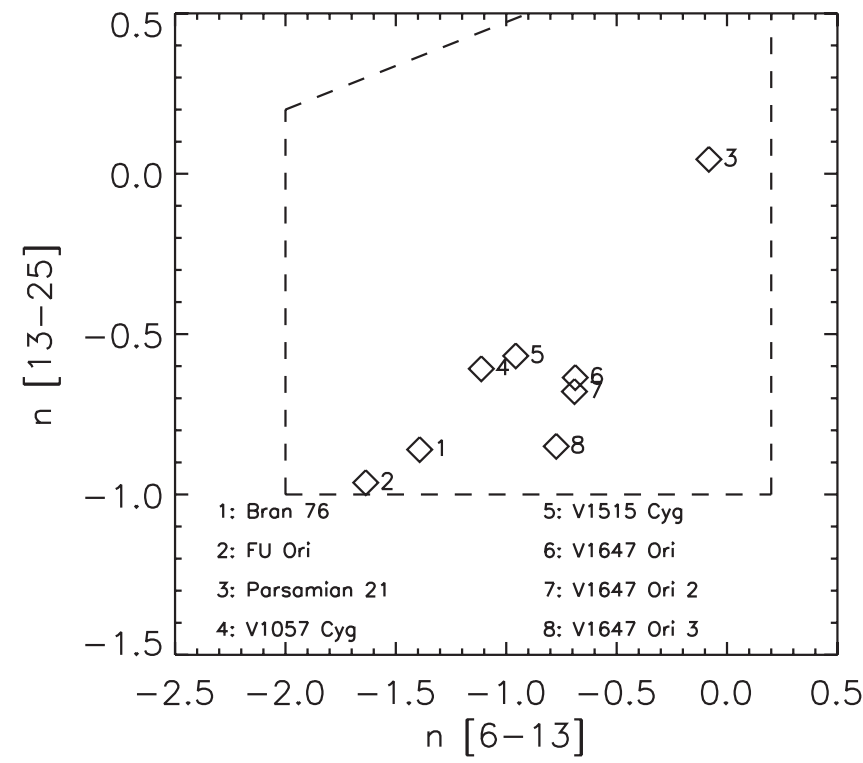

FIG. 23.- Spectral index $n$ of the silicate emission objects evaluated between 13 and $25 \mu \mathrm{m}$ vs. the spectral index between 6 and $13 \mu \mathrm{m} ; n$ is computed as $n \equiv$ $d \log \left(\lambda F_{\lambda}\right) / d \log (\lambda)$. All objects populate the same area in the plot as the Class II objects in Taurus presented by Furlan et al. (2006) (dashed area). Due to the steep rise in its SED, Parsamian 21 lies slightly off from the other objects.

feature of low-mass stars and the overall SEDs is similar to that of intermediate-mass stars: embedded objects, showing a pristine silicate band in absorption, evolve into objects showing a combination of silicate absorption and emission, and finally pure emission features appear, where dust processing leads to a broad range of shapes and strength. Since all these stages are represented in our sample, we believe that, indeed, the FUor phase is the link between the embedded Class I objects and the more evolved Class II objects. In this context, the objects showing silicate absorption are younger and at the beginning of the period where subsequently numerous FUor outbursts will occur, while the objects showing pure emission features are more evolved and possibly near the end of their FUor period. As illustrated in Figure 23, the spectral indices of the emission objects are indistinguishable from Class II objects in Taurus (Furlan et al. 2006).
In particular, the objects FU Ori and Bran 76 appear to be the most evolved objects because, as mentioned above, their SEDs do not require the presence of a large remnant envelope. A nice example of an intermediate object is RNO 1B, where the dominating absorption feature is altered by the underlying silicate emission from the accretion disk. Figure 24 illustrates with simple sketches the main features of the two categories of FUors. It should be mentioned that theoretically a Class II FUor seen close to edgeon might be interpreted as a Class I FUor. As outlined in $\S$ 4.1.1, it is, however, statistically very unlikely that all of the Class I objects shown here are Class II FUors "in disguise."

One question arises, however. If FUors are younger than classical T Tauri stars but already show clear evidence for dust coagulation, then why do some classical T Tauri stars show silicate emission features consisting purely of pristine and unprocessed dust? One possible explanation is linked to the high accretion rates of the FUors. It is believed that high disk accretion rates are related to a higher degree of turbulence and thus mixing in the disk. As a result, larger particles are coupled to the turbulent gas and mixed throughout the disk and to the disk surface, where they can be observed. Once the accretion rate, and hence the amount of mixing, drops, larger particles tend to settle to the disk midplane much faster than small particles, which remain in the disk surface layer. Thus, although large particles are present in the disk of a T Tauri star, they might not reach the disk surface, and only the small grains produce a silicate emission feature. Again, as for the issue concerning the lack of crystalline grains, a more detailed theoretical modeling is certainly required to see to what extent this qualitative explanation is valid.

\subsection{Parsamian 21: Intermediate-Mass FUor or Post-AGB Star?}

The strong PAH emission of Parsamian 21 is unique among the sample of FUors presented here, and the analysis raises serious doubts about whether Parsamian 21 is indeed a member of this group. The initial classification was made by Staude \& Neckel (1992) based on optical spectroscopy. These authors derived a spectral type of F5 Iab, found a prominent P Cygni profile in $\mathrm{H} \alpha$, and observed shock-induced emission in [O $\mathrm{I}],[\mathrm{N} \mathrm{II]}$, and $[\mathrm{S}$ II]. They also mention the detection of $\mathrm{Li}$ in their spectra, which, if present, does not seem to be significantly above the

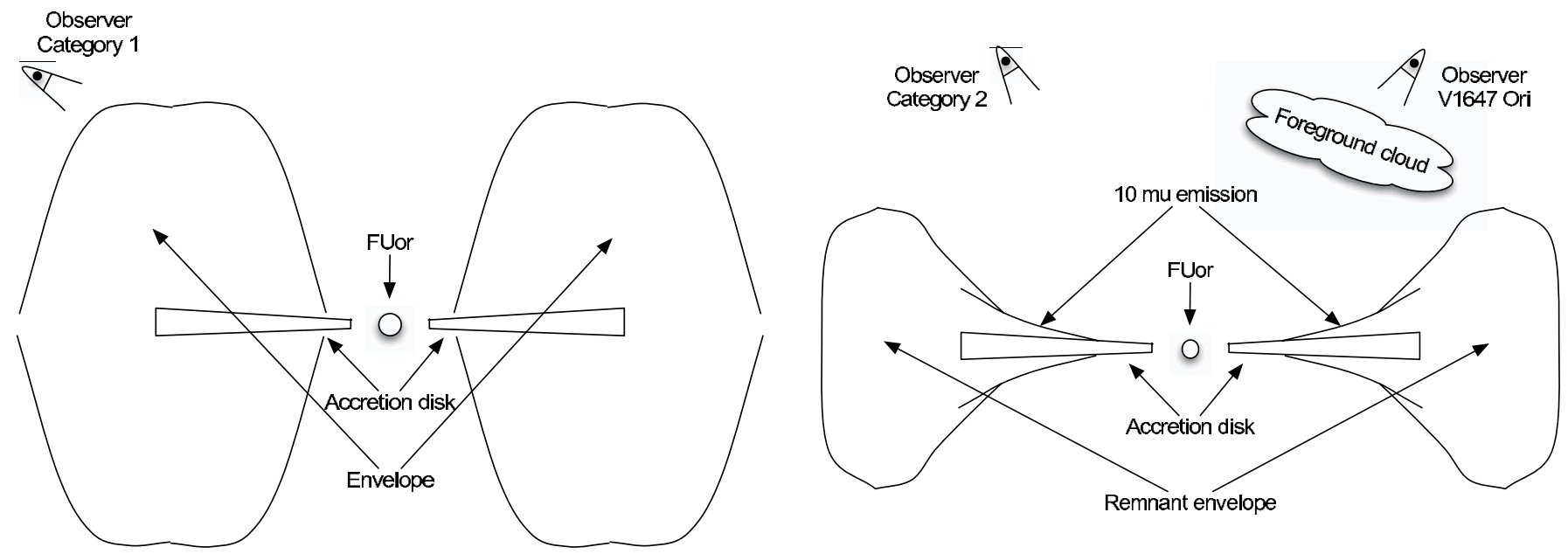

FIG. 24.- Simple graphical representation explaining the two categories of FUors (not to scale). Category 1 objects (left) show silicate absorption and are younger than Category 2 objects (right), which show silicate emission. While Category 1 objects are still more deeply embedded in their envelopes and appear to be Class I sources, Category 2 FUors are similar to Class II sources. The spectrum of V1647 Ori can be explained assuming that extinction in the line of sight to this object creates the ice absorption features and reduces also the strength of the observed silicate emission. 
noise level in the data. New observations with higher spectral resolution and higher signal-to-noise ratio would certainly be eligible. Although some of these features are commonly observed in FUors, they are not unique to this group of objects, as also HAeBes show, for instance, $\mathrm{P}$ Cygni profiles in $\mathrm{H} \alpha$. In addition, two of the main properties of FUors, an outburst in optical light and $\mathrm{CO}$ band head absorption profiles in the NIR (e.g., Hartmann \& Kenyon 1996), have not yet been observed for Parsamian 21. Looking at the immediate surroundings of Parsamian 21 in optical data from the Digitized Sky Survey, ${ }^{14}$ it is also striking that no dark cloud complex is linked to Parsamian 21, and hence no connection to a larger star-forming region is present.

To excite the PAH emission, sufficiently strong UV radiation is required. However, most FUors have a later spectral type than Parsamian 21 and in general lack, unlike T Tauri stars, UV continuum excess emission (Hartmann \& Kenyon 1996).

As mentioned above, the positions of the PAH emission bands are atypical for young stars, and so far only seven objects are known to show characteristics of PAH class C objects (Sloan et al. 2007) as defined by Peeters et al. (2002). Among these seven objects, only two presumably young stars (SU Aur and HD 135344) show the peculiar PAH band close to $8.2 \mu \mathrm{m}$. All other PAH class C objects are post-AGB stars or red giants (Sloan et al. 2007; Peeters et al. 2002).

In addition, one of the post-AGB stars from Peeters et al. (2002) and also HD 56126, a post-AGB star with PAH and silicate emission (Hony et al. 2003), have the same spectral type as Parsamian 21. However, we should mention that Dibai (1969) and The et al. (1994) give a spectral type of A5 Ve $\alpha$ for Parsamian 21. This is too early for a typical FU Orionis object but could also explain the PAH emission. Finally, the bipolar emission knots seen in $\mathrm{H} \alpha$ and [ $\mathrm{N}_{\mathrm{II}}$ ] in the optical spectrum of Parsamian 21 (Staude \& Neckel 1992) are also found in the bipolar outflows of evolved stars and "preplanetary" nebulae (e.g., in the Butterfly Nebula; Solf 2000). They are thus not only seen in young objects.

Based on these findings, we believe that the FUor status of Parsamian 21 is at least very questionable. Either this object represents an intermediate-mass FUor, suggesting that also stars in this mass regime undergo phases of enhanced accretion, or, and this appears even more likely, Parsamian 21 is not even a young object but an evolved star, sharing typical properties with postAGB stars.

\section{CONCLUSIONS AND FUTURE PROSPECTS}

Our conclusions can be summarized as follows:

1. We presented the first coherent space-based spectroscopic MIR study of 14 FUors observed with the Spitzer Space Telescope or the Infrared Space Observatory. The sample includes roughly two-thirds of the known FUors or FUor candidates.

2. Based on the appearance of the $10 \mu \mathrm{m}$ silicate feature, we divided the sample into two categories: Category 1 objects show the silicate feature in absorption, and the spectra show additional absorption bands at 6.0, 6.8, and $15.2 \mu \mathrm{m}$ due to ices. Category 2 objects show silicate emission and (most of them also) indications for water vapor absorption at shorter wavelengths. Only one Category 2 object (V1647 Ori) shows ice absorption bands, which we explain by foreground extinction and an intrinsically strong $10 \mu \mathrm{m}$ emission feature.

3. The silicate absorption is best explained with dusty and icy envelopes surrounding the Category 1 objects. Statistical reasons argue against all objects having accretion disks seen edge-on.

14 See http://archive.eso.org/dss/dss.
The silicate emission of the Category 2 FUors arises from the surface layer of the surrounding accretion disks.

4. The shape of the silicate band of the Category 1 objects is in agreement with typical dust compositions of the ISM. For one object (RNO 1B) the shape of the feature and the decrease in optical depth longward of $10 \mu \mathrm{m}$ can be explained with a superposed emission feature.

5. Optical depths for the observed silicate and ice absorption bands were derived. We find an apparent correlation among the optical depths of the ices, indicating similar environmental conditions for the objects. For the silicate feature, no correlation is expected.

6. Using different extinction curves, we computed the optical extinction toward the objects based on the depth of the $10 \mu \mathrm{m}$ silicate features. The results are in agreement with values found in the literature, given the uncertainties in the dust models and for the optical extinction values in the references. For RNO $1 \mathrm{~B}$ the derived value for $A_{V}$ is smaller than expected, indicating that on-top silicate emission might influence the optical depth at $10 \mu \mathrm{m}$.

7. The emission profiles of the Category 2 objects show clear evidence for grain growth. Fitting a dust model to the spectrum of FU Ori reveals that, indeed, larger grains than typically observed in the ISM are required to explain the shape. As FUors are presumably younger than T Tauri stars (see below), this indicates that grain growth sets in very early during disk evolution.

8. Despite the high accretion rates of the FUor accretion disks and the resulting higher disk temperatures and mixing rates, we find hardly any evidence for crystalline grains. So far we lack a clear explanation for these observational results and leave it to further investigations.

9. The two categories of FUors can be explained within a single paradigm, where Category 1 objects are younger and similar to Class I objects, while Category 2 FUors are more evolved and show already properties of Class II sources. This explanation is in agreement with theoretical models that expect FUors to undergo several outbursts before they enter the more quiescent classical T Tauri phase. Thus, the FUor phase might indeed be the link between Class I and Class II objects and common to most young low-mass stars.

10. For OO Ser and V1647 Ori the multiepoch data allow an analysis of the postoutburst fading of the objects. Both objects faded significantly over timescales of a few months, suggesting that the outbursts might be intermediate between the long-lived FUor eruptions and the short-lived EXor-type events.

11. Only one object (Parsamian 21) shows PAH emission similar to that often observed in post-AGB stars. We find that most other observational data for Parsamian 21 can also be explained by the object being an evolved star. In consequence, the FUor status for this object is questioned.

Based on these findings, future investigations might include the following points:

1. A complete MIR spectroscopic census of all known FUors would complement the data presented here and might help to derive conclusions concerning the duration of the FUor phase using statistical arguments.

2. Multidimensional radiative transfer models have not yet been applied to FUor accretion disks, but they are required to derive a coherent picture of the disk structure, including the emission layer.

3. Models for the dust evolution in accretion disks could try to explain quantitatively the observed large grain population and the apparent lack of crystalline silicates. 
4. Finally, if our conclusions are correct, then some of the known low-mass Class I objects might be FU Ori objects hidden in a quiescent phase between two consecutive outbursts. The observational properties of these objects might be revisited to search for any indication of FUor properties.

S. P. Q. kindly acknowledges support from the German Friedrich-Ebert-Stiftung. We are grateful to Henrik Beuther and
Kees Dullemond for interesting and insightful discussions and thank the referee for a detailed report helping to improve the style and content of this paper. The version of the $I S O$ data presented in this paper corresponds to Highly Processed Data Product (HPDP) sets available for public use in the ISO Data Archive. OSIA is a joint development of the SWS consortium. Contributing institutes are SRON, MPE, KUL, and the ESA Astrophysics Division. This research has made use of the SIMBAD database, operated at CDS, Strasbourg, France.

\section{Facilities: Spitzer, ISO}

Ábrahám, P., Mosoni, L., Henning, T., Kóspál, Á., Leinert, C., Quanz, S. P., \& Ratzka, T. 2006, A\&A, 449, L13

Acke, B., \& van den Ancker, M. E. 2004, A\&A, 426, 151

Acosta-Pulido, J. A., et al. 2007, AJ, 133, 2020

Bell, K. R., Cassen, P. M., Klahr, H. H., \& Henning, T. 1997, ApJ, 486, 372

Bergin, E. A., Melnick, G. J., Gerakines, P. A., Neufeld, D. A., \& Whittet, D. C. B. 2005, ApJ, 627, L33

Boley, A. C., Mejía, A. C., Durisen, R. H., Cai, K., Pickett, M. K., \& D'Alessio, P. 2006, ApJ, 651, 517

Boogert, A. C. A., et al. 2004, ApJS, 154, 359

Bouwman, J., Lawson, W. A., Dominik, C., Feigelson, E. D., Henning, T., Tielens, A. G. G. M., \& Waters, L. B. F. M. 2006, ApJ, 653, L57

Bouwman, J., Meeus, G., de Koter, A., Hony, S., Dominik, C., \& Waters, L. B. F. M. 2001, A\&A, 375, 950

Campbell, B., Persson, S. E., Strom, S. E., \& Grasdalen, G. L. 1988, AJ, 95, 1173

Casali, M. M. 1991, MNRAS, 248, 229

Close, L. M., Roddier, F., Northcott, M. J., Roddier, C., \& Graves, J. E. 1997, ApJ, 478, 766

Coffey, D., Downes, T. P., \& Ray, T. P. 2004, A\&A, 419, 593

Cohen, M., \& Kuhi, L. V. 1979, ApJS, 41, 743

Desch, S. J., \& Cuzzi, J. N. 2000, Icarus, 143, 87

Dibai, E. A. 1969, Astrophysics, 5, 115

Dorschner, J., Begemann, B., Henning, T., Jaeger, C., \& Mutschke, H. 1995, A\&A, 300, 503

Draine, B. T. 2003, ARA\&A, 41, 241

Ehrenfreund, P., Dartois, E., Demyk, K., \& D’Hendecourt, L. 1998, A\&A, 339, L17

Elia, D., Strafella, F., Campeggio, L., Giannini, T., Lorenzetti, D., Nisini, B., \& Pezzuto, S. 2004, ApJ, 601, 1000

Forrest, W. J., et al. 2004, ApJS, 154, 443

Fridlund, C. V. M., Bergman, P., White, G. J., Pilbratt, G. L., \& Tauber, J. A. 2002, A\&A, 382, 573

Furlan, E., et al. 2006, ApJS, 165, 568

Gail, H.-P. 2001, A\&A, 378, 192

Geers, V. C., et al. 2006, A\&A, 459, 545

Graham, J. A., \& Frogel, J. A. 1985, ApJ, 289, 331

Green, J. D., Hartmann, L., Calvet, N., Watson, D. M., Ibrahimov, M., Furlan, E., Sargent, B., \& Forrest, W. J. 2006, ApJ, 648, 1099

Hanner, M. S., Brooke, T. Y., \& Tokunaga, A. T. 1998, ApJ, 502, 871

Harker, D. E., \& Desch, S. J. 2002, ApJ, 565, L109

Hartmann, L., \& Kenyon, S. J. 1985, ApJ, 299, 462 1996, ARA\&A, 34, 207

Herbig, G. H. 1977, ApJ, 217, 693

Hony, S., Tielens, A. G. G. M., Waters, L. B. F. M., \& de Koter, A. 2003, A\&A, 402, 211

Keane, J. V., Tielens, A. G. G. M., Boogert, A. C. A., Schutte, W. A., \& Whittet, D. C. B. 2001, A\&A, 376, 254

Kemper, F., Vriend, W. J., \& Tielens, A. G. G. M. 2004, ApJ, 609, 826

Kessler-Silacci, J., et al. 2006, ApJ, 639, 275

Kessler-Silacci, J. E., Hillenbrand, L. A., Blake, G. A., \& Meyer, M. R. 2005, ApJ, 622, 404

Knez, C., et al. 2005, ApJ, 635, L145

Kóspál, Â., Ábrahám, P., Prusti, T., Acosta-Pulido, J., Hony, S., Moór, A., \& Siebenmorgen, R. 2007, A\&A, 470, 211
Lachaume, R. 2004, A\&A, 422, 171

Larsson, B., et al. 2000, A\&A, 363, 253

Levreault, R. M. 1988, ApJS, 67, 283

Lorenzetti, D., et al. 2000, A\&A, 357, 1035

Malbet, F., et al. 2005, A\&A, 437, 627

Mathis, J. S. 1990, ARA\&A, 28, 37

Meeus, G., Waters, L. B. F. M., Bouwman, J., van den Ancker, M. E., Waelkens, C., \& Malfait, K. 2001, A\&A, 365, 476

Men'shchikov, A. B., \& Henning, T. 1997, A\&A, 318, 879

Men'shchikov, A. B., Henning, T., \& Fischer, O. 1999, ApJ, 519, 257

Millan-Gabet, R., et al. 2006, ApJ, 641, 547

Muzerolle, J., Megeath, S. T., Flaherty, K. M., Gordon, K. D., Rieke, G. H., Young, E. T., \& Lada, C. J. 2005, ApJ, 620, L107

Peeters, E., Hony, S., Van Kerckhoven, C., Tielens, A. G. G. M., Allamandola, L. J., Hudgins, D. M., \& Bauschlicher, C. W. 2002, A\&A, 390, 1089

Pilipp, W., Hartquist, T. W., Morfill, G. E., \& Levy, E. H. 1998, A\&A, 331, 121

Polomski, E. F., et al. 2005, AJ, 129, 1035

Pontoppidan, K. M., Dullemond, C. P., van Dishoeck, E. F., Blake, G. A., Boogert, A. C. A., Evans, N. J., II, Kessler-Silacci, J. E., \& Lahuis, F. 2005, ApJ, 622, 463

Przygodda, F., van Boekel, R., Àbrahàm, P., Melnikov, S. Y., Waters, L. B. F. M., \& Leinert, C. 2003, A\&A, 412, L43

Quanz, S. P., Henning, T., Bouwman, J., Linz, H., \& Lahuis, F. 2007, ApJ, 658, 487

Quanz, S. P., Henning, T., Bouwman, J., Ratzka, T., \& Leinert, C. 2006, ApJ, 648,472

Reipurth, B., Hartmann, L., Kenyon, S. J., Smette, A., \& Bouchet, P. 2002, AJ, 124,2194

Sandell, G., \& Weintraub, D. A. 2001, ApJS, 134, 115

Sargent, B., et al. 2006, ApJ, 645, 395

Sato, S., Okita, K., Yamashita, T., Mizutani, K., Shiba, H., Kobayashi, Y., \& Takami, H. 1992, ApJ, 398, 273

Schutte, W. A., \& Khanna, R. K. 2003, A\&A, 398, 1049

Schütz, O., Meeus, G., \& Sterzik, M. F. 2005, A\&A, 431, 165

Skinner, S. L., Briggs, K. R., \& Güdel, M. 2006, ApJ, 643, 995

Sloan, G. C., et al. 2005, ApJ, 632, 956 2007, ApJ, 664, 1144

Smith, H. A., Fischer, J., Schwartz, P. R., \& Geballe, T. R. 1987, ApJ, 316, 265

Snell, R. L., \& Schloerb, F. P. 1985, ApJ, 295, 490

Solf, J. 2000, A\&A, 354, 674

Staude, H. J., \& Neckel, T. 1991, A\&A, 244, L13 1992, ApJ, 400, 556

Stocke, J. T., Hartigan, P. M., Strom, S. E., Strom, K. M., Anderson, E. R., Hartmann, L. W., \& Kenyon, S. J. 1988, ApJS, 68, 229

The, P. S., de Winter, D., \& Perez, M. R. 1994, A\&AS, 104, 315

van Boekel, R., Min, M., Waters, L. B. F. M., de Koter, A., Dominik, C., van den Ancker, M. E., \& Bouwman, J. 2005, A\&A, 437, 189

van Boekel, R., Waters, L. B. F. M., Dominik, C., Bouwman, J., de Koter, A., Dullemond, C. P., \& Paresce, F. 2003, A\&A, 400, L21

van Dishoeck, E. F. 2004, ARA\&A, 42, 119

Vorobyov, E. I., \& Basu, S. 2006, ApJ, 650, 956

Watson, D. M., et al. 2004, ApJS, 154, 391

Weingartner, J. C., \& Draine, B. T. 2001, ApJ, 548, 296

Weintraub, D. A., Sandell, G., \& Duncan, W. D. 1991, ApJ, 382, 270

White, G. J., et al. 2000, A\&A, 364, 741

White, R. J., \& Ghez, A. M. 2001, ApJ, 556, 265 